\title{
Activity-Dependent Modulation of the Interaction between CaMKII $\alpha$ and Abil and Its Involvement in Spine Maturation
}

\author{
Esther Park, ${ }^{1}$ Susan Chi, ${ }^{2}$ and Dongeun Park ${ }^{1}$ \\ ${ }^{1}$ School of Biological Sciences, Seoul National University, Seoul 151-747, South Korea, and ${ }^{2}$ Center for Basic Research in Digestive Diseases, Mayo Clinic, \\ Rochester, Minnesota 55905
}

Remodeling of dendritic spines through regulation of actin dynamics is a key event in activity-dependent structural plasticity. However, the molecular mechanism underlying this process is poorly understood. Here, we show that activity-dependent modulation of Abl interactor $1-\mathrm{Ca}^{2+} /$ calmodulin-dependent kinase II $\alpha$ (Abi1-CaMKII $\alpha$ ) interaction, and thereby their activity, is important for regulation of spine morphology in cultured rat hippocampal neurons. Abil interacts with CaMKII $\alpha$ at resting conditions through Abil's tSNARE (target membrane-associated SNARE), which harbors striking homology with CaMKII $\alpha$ regulatory domain. The interaction of the two proteins, Abil and CaMKII $\alpha$, results in their simultaneous inhibition, inhibition of CaMKII $\alpha$ activity, and also inhibition of Abildependent Rac activation. Their functional impediment is released when they dissociate from each other by calmodulin binding through glutamate receptor activation. Before dissociation, Abil is phosphorylated by CaMKII $\alpha$ at serine 88, which may involve in regulation of Rac activation and spine maturation. Our results suggest that modulation of the interaction between Abil and CaMKII $\alpha$, through the glutamate receptor pathway, may be a molecular mechanism underlying activity-regulated structural plasticity in rat hippocamapal neurons.

\section{Introduction}

Dendritic spines, which are small, highly specialized, and dynamic structures in neurons, receive and relay synaptic information in response to synaptic activity by undergoing rapid morphological alterations (Nimchinsky et al., 2002). Reorganization of the actin cytoskeleton, a major cytoskeleton in the synapse, is a key event in modulation of the structural modifications of excitatory synapses (Dillon and Goda, 2005; Cingolani and Goda, 2008).

$\mathrm{Ca}^{2+} /$ calmodulin-dependent kinase II (CaMKII) is a predominant serine/threonine kinase in the postsynaptic density of excitatory synapses and has been suggested to be a key molecule in regulating functional and structural synaptic plasticity. CaMKII activation is necessary and sufficient for induction of activitydependent spine plasticity (Maletic-Savatic et al., 1999; Toni et al., 1999; Matsuzaki et al., 2004). CaMKII also has been shown to control activity-dependent filopodial growth and branching, as well as spine formation ( $\mathrm{Wu}$ and Cline, 1998; Jourdain et al., 2003; Andersen et al., 2005).

Because of its switch-like properties and abundance in the brain, CaMKII has come to be considered a crucial enzyme in the

\footnotetext{
Received May 10, 2012; revised July 18, 2012; accepted July 22, 2012.

Author contributions: E.P. and D.P. designed research; E.P. and S.C. performed research; E.P. and D.P. analyzed data; E.P. and D.P. wrote the paper.

This work was supported by Grant M103KV010013-04K2201-01310 from the Brain Research Center, funded by the Korean Government, to D.P.

The authors declare no competing financial interests.

Correspondence should be addressed to Dongeun Park, Seoul National University, 599 Gwanak-ro, Gwanak-gu Building 504, Room 503, Seoul, South Korea 151-747. E-mail: depark@snu.ac.kr.

DOI:10.1523/JNEUROSCI.2257-12.2012

Copyright $\odot 2012$ the authors $\quad 0270-6474 / 12 / 3213177-12 \$ 15.00 / 0$
}

brain and CaMKII's regulatory mechanisms have been intensively studied. NMDA subunit (NR2B) and Drosophila Eag potassium channel have been found to recruit CaMKII to synapses and prolong CaMKII activity after $\mathrm{Ca}^{2+} / \mathrm{CaM}$ release from the enzyme (Bayer et al., 2001; Wang et al., 2002; Sun et al., 2004). Thus far, many groups have focused on the mechanisms for retention of CaMKII $\alpha$ activation. However, the intermolecular mechanisms of regulating basal CaMKII $\alpha$ activity have not been reported yet.

Abl interactor 1 (Abi1) acts as a cofactor essential for specifying Sos 1 guanine nucleotide exchange factor activity for Rac GTPase when in complex with Eps8 and PI3K (Scita et al., 1999; Innocenti et al., 2003). Also, Abil was recently reported to directly bind to Rac (Dubielecka et al.). In addition, the stability of WAVE1 and WAVE2 complexes which are important in regulation of Arp2/3 complexes, have also been reported to be mediated by Abil (Innocenti et al., 2003; Leng et al., 2005). Abi family proteins have also been implicated in neuronal morphogenesis. Overexpression and knockdown of Abil in hippocampal neurons at 3 and 10 DIV demonstrate an essential function of Abil in dendritic morphology (Proepper et al., 2007).

Here, we examined the roles of Abil and CaMKII $\alpha$ in structural plasticity and the molecular mechanisms underlying their regulation. We found that binding of Abil to CaMKII $\alpha$ inhibits its Thr 286 autophosphorylation, which is crucial for kinase activation. Abil inhibits CaMKII $\alpha$ by mimicking its autoinhibitory domain through sequence homology. This interaction is regulated in a glutamate-dependent manner in which $\mathrm{Ca}^{2+}$-loaded CaM disrupts Abi1-CaMKII $\alpha$ binding. In addition, Abil is phosphorylated by CaMKII $\alpha$ and this phosphorylation is essential for maturation of dendritic spines through Rac activation. These 
data not only provide new insights into the molecular mechanisms underlying glutamate-dependent structural plasticity in dendritic spines, but also indicate novel functions of tSNARE (target membrane-associated SNARE) domain in neurons.

\section{Materials and Methods}

Antibodies and reagents. Antibodies purchased were anti-CaMKII $\alpha$ (clone $\mathrm{CB} \alpha-2$, Zymed Laboratories), anti-Abil pAb (clone T-15, Santa Cruz Biotechnology), anti-Abil mAb (Medical \& Biological Laboratories), anti-GFP (Santa Cruz Biotechnology), anti-Flag (Sigma-Aldrich), anti-phospho-Thr 286 CaMKII $\alpha$ (Santa Cruz Biotechnology), anti-actin (Sigma-Aldrich), anti-Myc (Santa Cruz Biotechnology), anti-calmodulin (Millipore Bioscience Research Reagents), anti-HA (Sigma-Aldrich), anti-pSer (clone PSR45, Sigma-Aldrich), anti-Rac (BD Biosciences), anti-vGlutl (Synaptic Systems), anti-NMDAR (Millipore Bioscience Research Reagents), and anti-PSD95 (Affinity BioReagents). Reagents purchased were KN92, KN93, and ionomycin (Calbiochem).

DNA constructs. The murine Abil cDNA used in this study was cloned from the Matchmaker Mus musculus brain cDNA Library (Clontech Laboratories). It is identical to Mus musculus Abil, transcript variant 1 (GenBank Accession No. BC004657). The coding region of murine Abil was subcloned into pcDNA3.1/MycHis vector (Invitrogen), pEGFP-C1 (Clontech Laboratories), and pcDNA-HA vectors. Glutathione S-transferase (GST)-R84E, GST-S88A, GST-T96A, GST-TT113,114VA, GFP-Abi1 ${ }^{\text {S88A }}$, and GFP$\mathrm{Abi1}^{\mathrm{S} 88 \mathrm{D}}$ mutants were cloned using Stratagene Mutagenesis kit. GST83-114 construct was used as the template for GST-R83E, GST-S88A, GST-T96A, and GST-TT113,114VA. GFP-CaMKII $\alpha$ and GFP-CaMKII $\alpha$ containing T286D mutation constructs were kind gifts from T. Meyer (Stanford University). GFP-tagged CaMKII-deletion mutants were generated by using GFP-CaMKII $\alpha$ as the template and by inserting stop codons at the ends of each domain (stop codons at His 273 for GFP-Ca and Gly 315 for GFPCaRe) using a Stratagene Mutagenesis kit. GFP-CaMKII $\alpha^{\text {K42E, T286A }}$ mutant was generated using the GFP-CaMKII $\alpha^{\text {T286A }}$ construct as the template. Knockdown construct pSIREN-AbilshRNA was constructed using primers targeting nucleotides 135-141. The shRNA-resistant GFP-Abil (GFP-AbilshRNAresis) construct was generated using a Stratagene $\mathrm{Mu}$ tagenesis kit. Five consecutive amino acids targeted by Abil shRNA were mutated to a different codon translating the same amino acid. Mutagenesis of all constructs was confirmed by DNA sequencing. SEP-NR2B was provided by Robert Malinow (Addgene plasmid 23998).

Cell culture, transfection, and immunoblotting. HeLa cells were maintained in DMEM (Invitrogen) supplemented with $10 \%$ certified fetal bovine serum (c-FBS, Invitrogen) and penicillin-streptomycin (Invitrogen) in $5 \% \mathrm{CO}_{2}$ at $37^{\circ} \mathrm{C}$. For Abil-knockdown stable cell lines, HeLa cells were transfected with pSIREN-DsRed as control or pSIREN-DsRedAbilshRNA using LipofectAMINE ${ }^{\text {PLUS }}$ (Invitrogen). Cells stably expressing the vectors were selected by addition of $400 \mu \mathrm{g} / \mathrm{ml}$ of G418 (Sigma-Aldrich) in growth media. DsRed-expressing cells were collected using FACS. The cells were then cultured into single cell colonies, each giving rise to a stable cell line. Transfection of cells with mammalian expression vectors were performed with LipofectAMINE ${ }^{\text {PLUS }}$ (Invitrogen) according to the manufacturer's instructions. Cells were washed twice with PBS and lysed with SDS-lysis buffer (100 mm Tris, pH 6.8, 2\% SDS, and $10 \%$ glycerol). Protein concentration was determined with BCA reagent (Pierce). Equal amounts of protein were resolved by SDSPAGE and transferred to a PVDF membrane. Blots were blocked with 5\% skim milk in PBS-T $(0.1 \%$ Triton X-100 in PBS) for 30 min. The blots were incubated with primary antibodies for $1 \mathrm{~h}$ and washed with PBS-T. Then the blots were incubated with horseradish peroxidase-conjugated secondary antibodies (Jackson ImmunoResearch Laboratories) and analyzed by enhanced chemiluminenesence.

GST pull-down assays and immunoprecipitation. GST pull-down assays were performed with GST fusion proteins expressed in E. coli (BL21) cells and purified by glutathione Sepharose beads (GE Healthcare Pharamia Biotech). The bound fusion proteins were then eluted by glutathione elution buffer (20 mM glutathione, $100 \mathrm{~mm}$ Tris- $\mathrm{HCl}, \mathrm{pH} 8.0,120 \mathrm{~mm}$ $\mathrm{NaCl}$ ). Purified proteins were quantified by Bradford quantification method (Bio-Rad). For isolation of GST-tSNARE-binding proteins, four brains of 4-week-old male mice (strain C57BL/6) were homogenized in 8 $\mathrm{ml}$ of homogenization buffer (50 mм Tris- $\mathrm{HCl}$, pH 8.5, 1 mм EDTA, 1 mM EGTA, $150 \mathrm{~mm} \mathrm{NaCl}, 2 \mathrm{~mm} \mathrm{Na}_{3} \mathrm{VO}_{4}, 15 \mathrm{~mm} \mathrm{NaF}, 1 \mu \mathrm{l} / \mathrm{ml}$ leupeptin, and $1 \mu \mathrm{l} / \mathrm{ml}$ pepstatin) with a Polytron homogenizer and incubated at $4^{\circ} \mathrm{C}$ for $1 \mathrm{~h}$ after addition of $1 \%$ Triton $\mathrm{X}-100$. The homogenates were centrifuged at $15,000 \mathrm{rpm}$ for $30 \mathrm{~min}$ at $4^{\circ} \mathrm{C}$. The supernatant was used as brain lysate for GST pull-down assays. One milligram of brain lysate was incubated with $4 \mu \mathrm{g}$ of GST or GST-tSNARE for $2 \mathrm{~h}$ at $4^{\circ} \mathrm{C}$ in immunoprecipitation buffer (50 mM Tris-HCl, pH 7.4, 1\% Triton X-100, $150 \mathrm{~mm}$ $\mathrm{NaCl}, 15 \mathrm{~mm} \mathrm{NaF}, 1 \mu \mathrm{l} / \mathrm{ml}$ leupeptin, $1 \mu \mathrm{l} / \mathrm{ml}$ pepstatin, and $1 \mu \mathrm{l} / \mathrm{ml}$ aprotinin). Glutathione Sepharose beads were added to the samples for $1 \mathrm{~h}$ at $4^{\circ} \mathrm{C}$ and washed with immunoprecipitation buffer three times to remove unbound proteins. Proteins bound to beads were denatured in Laemelli sample buffer and subjected to SDS-PAGE and Western blotting. Fourteen days in vitro rat cortical neurons were incubated in Tyrode solution (119 mM NaCl, $2.5 \mathrm{~mm} \mathrm{KCl}, 2 \mathrm{~mm} \mathrm{CaCl}, 2 \mathrm{~mm} \mathrm{MgCl} 2,25 \mathrm{~mm}$ HEPES, pH 7.4, and $30 \mathrm{~mm}$ glucose) for $30 \mathrm{~min}$ before treatment with 50 $\mu \mathrm{M}$ glutamate and $5 \mu \mathrm{M}$ glycine for $5 \mathrm{~min}$.

For in vitro binding assays, $30 \mathrm{ng}$ of purified rat brain CaMKII (Calbiochem) was incubated for $10 \mathrm{~min}$ at $30^{\circ} \mathrm{C}$ with or without $0.3 \mathrm{~mm}$ $\mathrm{Ca}^{2+}, 2 \mu \mathrm{M} \mathrm{CaM}$, and $1 \mu \mathrm{M}$ ATP in kinase buffer (50 mM HEPES, pH 7.4, and $5 \mathrm{~mm} \mathrm{MgCl}_{2}$ ). Then CaMKII, loaded with $\mathrm{Ca}^{2+} / \mathrm{CaM}$ and/or ATP, was mixed with $2 \mu \mathrm{g}$ of GST-47-114 and incubated for $1 \mathrm{~h}$ at $4^{\circ} \mathrm{C}$ in pull-down buffer (50 mм HEPES, pH 7.4, $5 \mathrm{~mm} \mathrm{MgCl}_{2}, 1 \%$ Triton X-100, $150 \mathrm{~mm} \mathrm{NaCl}, 15 \mathrm{~mm} \mathrm{NaF}, 1 \mu \mathrm{g} / \mathrm{ml}$ leupeptin, $1 \mu \mathrm{g} / \mathrm{ml}$ aprotinin, and 1 $\mu \mathrm{g} / \mathrm{ml}$ pepstatin). Glutathione beads were then added and incubated for $1 \mathrm{~h}$ at $4^{\circ} \mathrm{C}$. The beads were then washed and the bead-bound proteins were separated by SDS-PAGE and immunoblotted for CaMKII $\alpha$ and phospho-Thr 286-CaMKII $\alpha$. The fusion proteins were visualized by Coomassie Blue staining.

Neuron culture and transfection. Rat hippocampal and cortical neuron cultures were prepared from embryonic day 18 Sprague Dawley rat embryos of either sex as previously described (Chang and De Camilli, 2001). Dissociated hippocampi and cortex tissues were treated with papain $(20$ $\mu \mathrm{g} / \mathrm{ml})$ and DNase $(10 \mathrm{U} / \mu \mathrm{l})$ for $40 \mathrm{~min}$ at $37^{\circ} \mathrm{C}$. The tissues were then mechanically dissociated by titration with a glass Pasteur pipette. Hippocampal neurons $\left(2 \times 10^{5}\right.$ cells $/ 60 \mathrm{~mm}$ dish $)$ and cortical neurons $(2 \times$ $10^{5}$ cells $/ \mathrm{ml}$ ) were plated in MEM (JBI) supplemented with $0.6 \%$ glucose, $1 \mathrm{~mm}$ sodium pyruvate, $1 \%$ penicillin-streptomycin (Invitrogen), 2 mM L-glutamine, and 10\% certified FBS (Invitrogen) for $4 \mathrm{~h}$ before exchange with Neurobasal medium (Invitrogen) containing $0.5 \mathrm{~mm}$ L-glutamine and B27 supplement (Invitrogen). The cells were maintained in a $5 \% \mathrm{CO}_{2}$ incubator at $37^{\circ} \mathrm{C}$ for up to $21 \mathrm{~d}$. Every $4-7 \mathrm{~d}$, half of the original media was discarded and replaced with fresh Neurobasal media supplemented with $0.5 \mathrm{~mm}$ glutamine and B27 supplement. Hippocampal neurons were transiently transfected at 6-8 DIV with a modified calcium phosphate method using CalPhos Transfection Kit (Calbiochem) (Ryan et al., 2005). Original media was exchanged with fresh Neurobasal media containing 25 mм HEPES, pH 7.35; 148 mм $\mathrm{CaCl}_{2}$ was mixed with $10 \mu \mathrm{g}$ of DNA; and distilled water was added dropwise to the mixture with vortexing, making a final volume of $100 \mu \mathrm{l}$. This mixture was then added dropwise to $100 \mu$ l of $2 \times$ HEPES-buffered solution while vortexing. The solution was left at room temperature for 2 min and then added dropwise to each $60 \mathrm{~mm}$ dish. Precipitate formed within $15 \mathrm{~min}$. After formation of precipitate, the cells were washed twice with fresh Neurobasal media and then incubated for $5 \mathrm{~min}$ at $37^{\circ} \mathrm{C}$. Following two more subsequent washings, the cells were returned to their original media.

Immunocytochemistry. Rat hippocampal neurons at 14 DIV seeded on $18 \mathrm{~mm}$ coverslips were fixed for $15 \mathrm{~min}$ in fixative (4\% paraformaldehyde and $4 \%$ sucrose in PBS). The neurons were permeabilized with $0.5 \%$ Triton X-100 in PBS for $10 \mathrm{~min}$ and then incubated in blocking solution [10\% c-FBS (Invitrogen), $0.5 \%$ gelatin, $0.1 \%$ Triton X-100 in PBS] for $30 \mathrm{~min}$. The coverslips were then incubated with primary antibodies diluted in blocking solution for $1 \mathrm{~h}$ at room temperature. After washing with PBS-T, coverslips were stained with fluorescein isothiocyanate-conjugated or tetramethyl rhodamine isothiocyanateconjugated anti-mouse or anti-rabbit IgG (Jackson ImmunoResearch Laboratories) for $1 \mathrm{~h}$. Following incubation, coverslips were washed with 
A

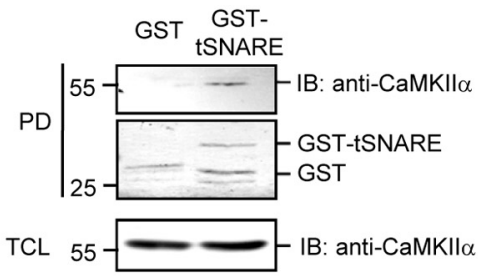

C

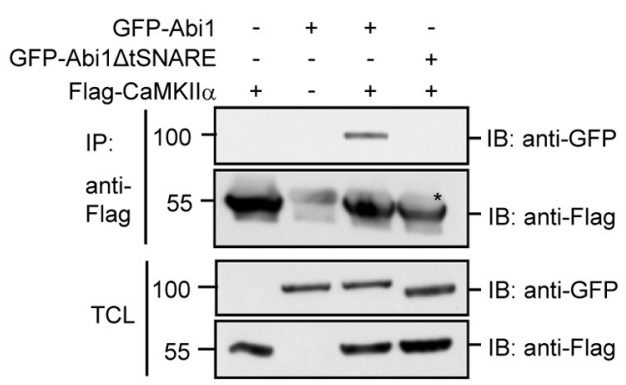

$\mathbf{E}$
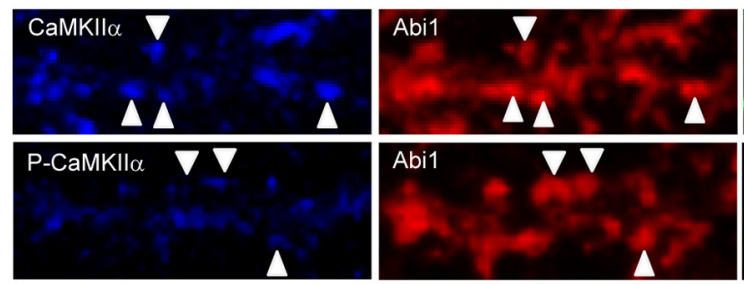

B

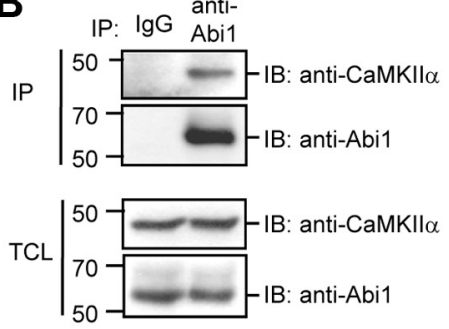

D

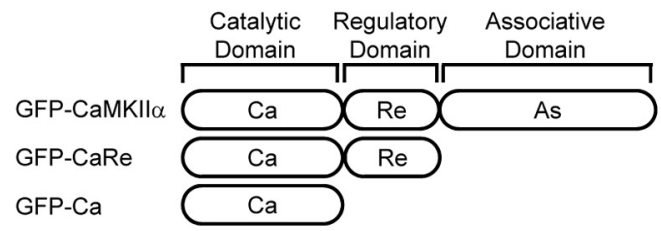

GFP-CaMKIl $\quad$ - WT CaRe Ca
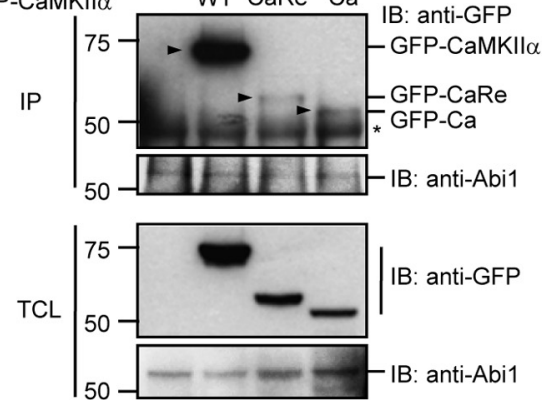

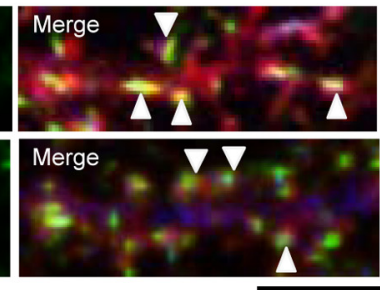

Figure 1. Abi1 tSNARE domain interacts with CaMKIII $\alpha$ catalytic domain. $\boldsymbol{A}$, Mouse brain lysates were incubated with purified GST or GST-tSNARE protein, and the bound proteins to GST fusion proteins were pulled-down (PD) by glutathione Sepharose beads and immunoblotted (IB) with anti-CaMKII $\alpha$ antibodies. GST fusion proteins were visualized by Coomassie Blue staining. $\boldsymbol{B}$, Lysates from 14 DIV rat cortical neurons were immunoprecipitated (IP) with control polyclonal lgGs or anti-Abi1 antibodies (anti-Abi1) and immunoblotted with anti-CaMKII $\alpha$ antibodies and anti-Abi1 antibodies. C, Lysates from HeLa cells expressing Flag-CaMKIl $\alpha$ and either GFP-Abi1 or GFP-Abi1 $\Delta$ tSNARE were immunoprecipitated with anti-Abi1 antibodies and immunoblotted with anti-GFP antibodies and anti-Flag antibodies. D, Lysates from HeLa cells expressing GFP-CaMKIll $\alpha$ (wild-type), GFP-CaRe (catalytic domain and regulatory domain), or GFP-Ca (catalytic domain) were immunoprecipitated with anti-Abi1 antibodies and immunoblotted with anti-GFP antibodies and anti-Abi1 antibodies. Arrowheads $(-)$ indicate coimmunoprecipitated CaMKII $\alpha$ proteins with Abi1. Asterisks in $\boldsymbol{C}$ and $\boldsymbol{D}$ indicate heavy chains of $\mathrm{g} \mathrm{G}$ s in the immunoprecipitates. $\boldsymbol{E}$, Immunocytochemistry of $14 \mathrm{DIV}$ rat hippocampal neurons in the top panels shows partial colocalization of the endogenous Abi1 (red) and CaMKII $\alpha$ (blue) at synapses visualized with anti-vGlut1 antibodies (green). Bottom panels show P-CaMKIl $\alpha$ (blue) localization with Abi1 (red) and vGlut1 (green). White arrowheads indicate regions of vGlut1 staining in each panel. Scale bar, $10 \mu \mathrm{m}$. TCL, Total cell lysate.

PBS-T, then mounted and observed with a Zeiss Axiovert fluorescence microscope equipped with a $100 \times, 1.4$ NA plan-apochromat objective lens (see Figs. $4 C, 7 C$ ) or with a Zeiss LSM710 confocal microscope equipped with $40 \times, 1.20$ NA C-Apochromat objective (see Fig. $1 E$ ). Where indicated, cells were treated with $10 \mu \mathrm{M}$ KN93 in Tyrode solution for $30 \mathrm{~min}$ before fixation.

Analysis of neuronal morphology and imaging. After image acquisition, images were cropped and processed using Adobe Photoshop. Image processing for colocalization assay was performed using colocalization plugin in Image (NIH) software. For morphometric analysis of neurons, protrusion length and spine width were measured using Axiovision (Zeiss) software. All morphometric experiments were repeated in at least three independent experiments with an $n>7$ per condition in each experiment. Protrusion lengths were defined as the length from the base of the neck to the farthest end of the spine head. Protrusions were classified into spines and filopodia as previously described (Zhang and Macara, 2006). Filopodia were defined as thin protrusions without a distinguishable head, and spines were defined as protrusions with a distinguishable head. Spine heads were defined as those with head/neck ratio of $>1.2$. Spine head widths were measured as the spine diameter perpendicular to the spine neck. Protrusion tip/shaft fluorescence intensity ratio of Thr 286 autophosphorylated CaMKII $\alpha$ (P-CaMKII $\alpha$ ) was analyzed by line-scan analyses using ImageJ software. A one-pixel wide line was drawn across the protrusion into the dendritic shaft and the fluorescence intensity profile along the line was measured and profiles were transferred to Microsoft Excel. To quantify protrusion tip/shaft fluorescence intensity ratio, the mean fluorescence intensities of protrusion tips and shafts were measured.

In vitro kinase assay. For phosphorylation assays, $1 \mathrm{ng}$ of purified rat brain CaMKII (Calbiochem) was activated in buffer containing $50 \mathrm{~mm}$ HEPES, pH 7.4, $5 \mathrm{~mm} \mathrm{MgCl}_{2}, 2 \mu \mathrm{M} \mathrm{CaM}, 0.3 \mathrm{mM} \mathrm{CaCl}_{2}$, and $0.5 \mathrm{~mm}$ $\left[\gamma_{-}{ }^{32} \mathrm{P}\right]$ ATP $(10 \mathrm{Ci} / \mathrm{mmol})$ for $10 \mathrm{~min}$ at $30^{\circ} \mathrm{C}$. As the control, the same buffer, but without $2 \mu \mathrm{M} \mathrm{CaM}$ and $0.3 \mathrm{mM} \mathrm{CaCl}_{2}$, was used. The reactions were stopped on ice and then incubated with $4 \mu \mathrm{g}$ of GST fusion proteins for $30 \mathrm{~min}$ at $30^{\circ} \mathrm{C}$. The reactions were then stopped with $5 \times$ Laemelli sample buffer and subjected to SDS-PAGE. The acrylamide gels were stained with Coomassie Blue staining solution to observe the total amount of GST fusion proteins and then dried on $3 \mathrm{M}$ Whatman paper. 

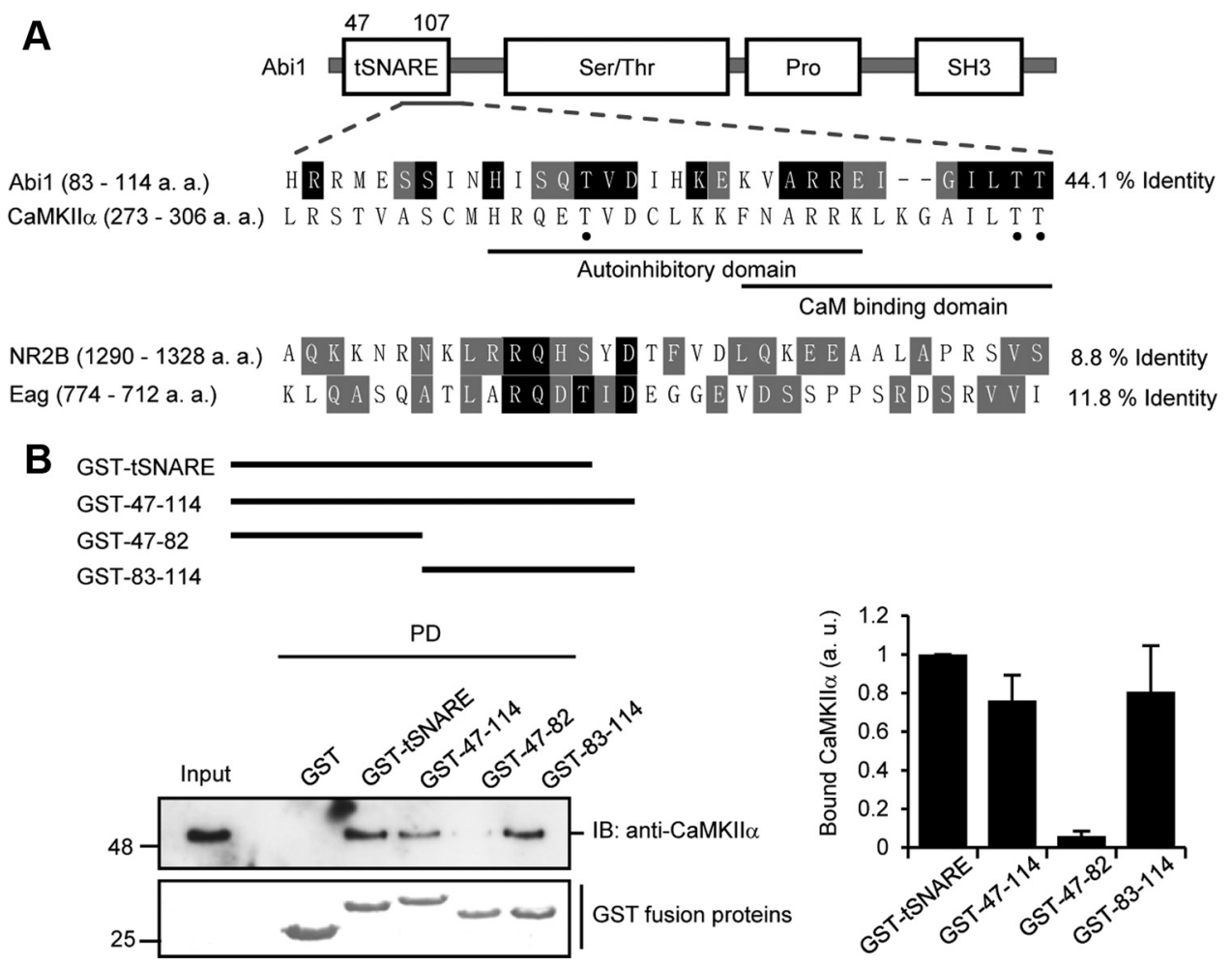

Figure 2. Abi1 tSNARE has high homology to CaMKII $\alpha$ regulatory domain and mediates Abi1 binding to CaMKIIl $\alpha$. A, Schematic representation of Abi1 and sequence alignment of Abi1, NR2B, and Eag channel to CaMKII $\alpha$. P-CaMKII $\alpha$ sites are indicated with black dot. Amino acids conserved between CaMKII $\alpha$ and other proteins are highlighted in black, and conservative substitutions are in gray. $\boldsymbol{B}$, Top, Schematic diagram of GST-tSNARE deletion mutants. Middle, In vitro GST pull-down (PD) assay. Purified rat brain CaMKII were incubated with indicated GST fusion proteins and analyzed for binding to CaMKII by immunoblotting (IB). GST fusion proteins were visualized by Coomassie Blue staining. Bottom, Results from three independent experiments were quantified, normalized to amount of GST fusion proteins, and expressed in arbitrary units (a. u.). Pro, Proline-rich domain.

Exposure of the gel to film showed phosphorylation of the GST fusion proteins.

Statistics. All analyses were completed from a minimum of three independent experiments. Statistical significances for measurements were calculated using Student's $t$ tests compared with control cells unless indicated. The actual $p$ values are indicated in the figure legends. All histograms representing Western blots are represented as mean \pm SD and all histograms analyzing morphogenic changes in neurons are represented as mean \pm SEM.

\section{Results}

\section{Abil tSNARE binds to CaMKII $\alpha$ catalytic domain}

Abil contains a tSNARE domain (Fig. 2 A) generally known to be involved in membrane fusion (Echarri et al., 2004). This domain has been reported to bind to Syntaxin-1 (Echarri et al., 2004) and to be involved in regulating nuclear shuttling of Abil (Proepper et al., 2007). Its function, however, remains elusive. To investigate novel pathways involving the tSNARE domain of Abil, we searched for binding partners to this domain in brain lysates of 4-week-old mice (strain C57BL/6) using a GST fusion protein of the Abil tSNARE domain (GST-tSNARE) as bait. Protein bands specific for GST-tSNARE binding were excised and subjected to matrix-assisted laser desorption ionization time-of-flight analysis (data not shown). One of the bands identified was CaMKII $\alpha$. We confirmed this interaction by GST pull-down assay (Fig. $1 A$ ). This binding was also corroborated in 14 DIV rat cortical neurons by coimmunoprecipitation with anti-Abil antibody (Fig. $1 B)$. Transient overexpression of wild-type GFP-Abil and tSNARE deletion mutant of Abil (GFP-Abi1 $\Delta$ tSNARE) in HeLa cells shows binding of CaMKII $\alpha$ to only GFP-Abi1, verifying that their interaction is through the tSNARE (Fig. 1C). Abil binds to
CaMKII $\alpha$ through its catalytic domain (Fig. 1D). Although wildtype CaMKII $\alpha$ was more efficiently coimmunoprecipitated with Abil than the deletion mutants, coimmunoprecipitation still took place, an indication that the presence of CaMKII $\alpha$ 's catalytic domain represents the minimum requirement for CaMKII $\alpha$ and Abil to bind CaMKII $\alpha$ is known to oligomerize into a dodecamer through its association domain (Rosenberg et al., 2005). Due to this oligomerization, wild-type CaMKII $\alpha$ would be more efficiently sedimented with Abil in comparison to mutants lacking the association domain. Comparison of the other two deletion mutants shows that Abil seems to bind with higher affinity to the deletion mutant comprised of only its catalytic domain (GFP$\mathrm{Ca}$. Colocalization of endogenous Abil and CaMKII $\alpha$ was also observed in 14 DIV rat hippocampal neurons (Fig. 1 E). Colocalization was observed in not all synapses but in a subset of synapses. Arrowheads indicate excitatory synapses identified by antivGlut1 immunostaining. The top panels in Figure $1 E$ show CaMKII $\alpha$ and Abil localization while the bottom panels show localization of Abi1 with P-CaMKII $\alpha$. Strong correlation of Abil and CaMKII $\alpha$ localization is seen in synapses. However, $\mathrm{P}-\mathrm{CaMKII} \alpha$ shows weak correlation with Abil localization. Notably, colocalization of CaMKII $\alpha$ and Abil is not seen in the dendritic shafts.

\section{Abil tSNARE domain has homology with CaMKII $\alpha$ regulatory domain}

In studying the interaction of the two proteins, Abil and CaMKII $\alpha$, sequence analyses showed a striking similarity between the Abil tSNARE domain and the CaMKII $\alpha$ regulatory domain. In a region of the Abil tSNARE domain (amino acids 
A
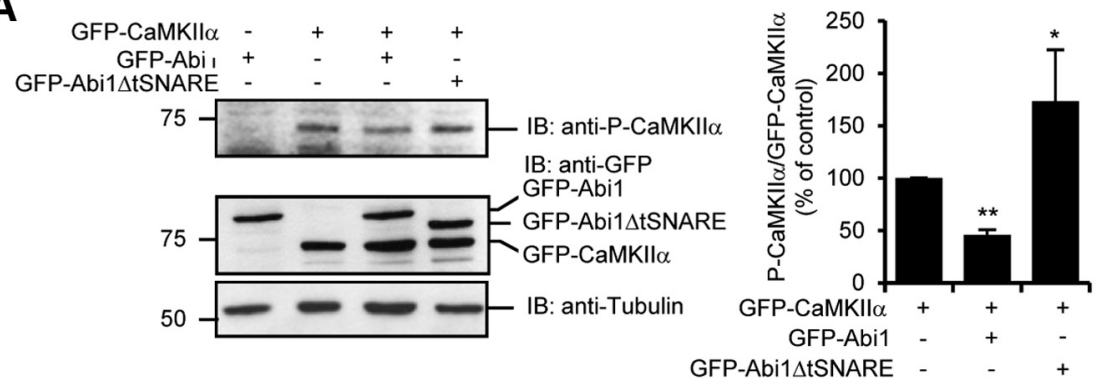

B

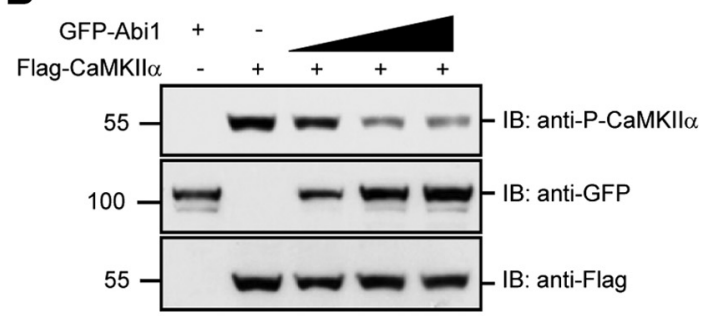

C
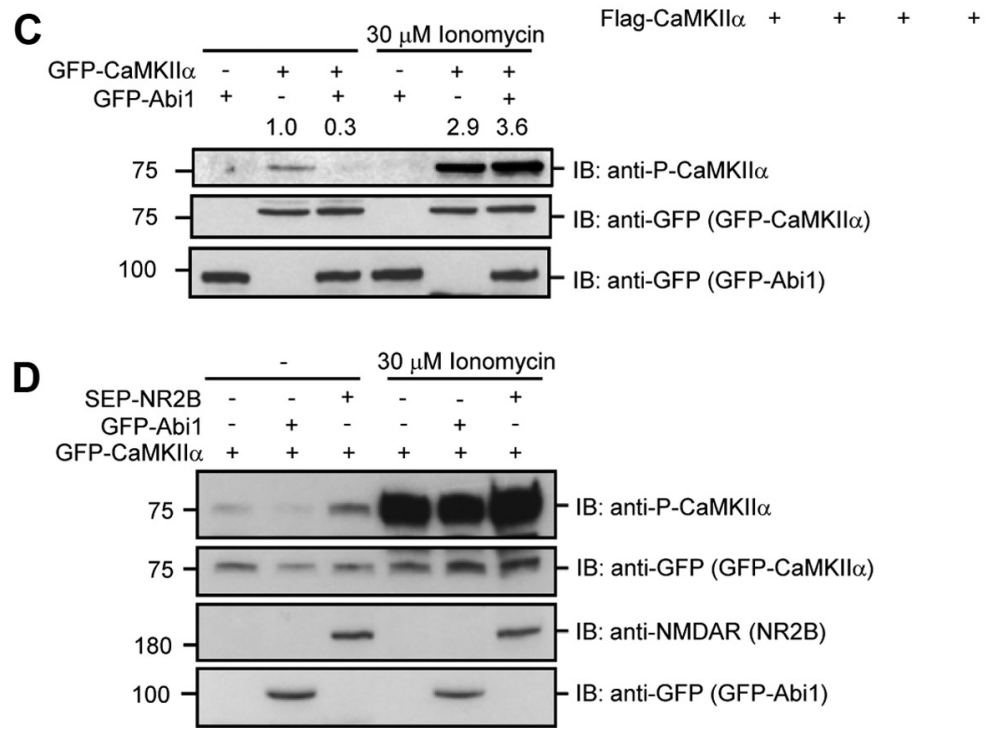

Figure 3. Binding of Abi1 inhibits P-CaMKII $\alpha$. A, Levels of CaMKII $\alpha$ Thr 286 autophosphorylation were examined by immunoblotting (IB) with anti-phospho-Thr 286 CaMKII $\alpha$ antibodies (P-CaMKII $\alpha$ ) in HeLa cells transfected with indicated constructs. Results from three independent experiments were quantified, normalized to GFP-CaMKII $\alpha$, and expressed as percentage of the amount in control cells (mean $\pm S D,{ }^{*} p=0.003,{ }^{* *} p=0.00003$, Student's $t$ test). $\boldsymbol{B}$, HeLa cells were transfected with the increasing levels of GFP-Abi1. Anti-Flag blot was used as the loading control. Results from three independent experiments were quantified, normalized to Flag-CaMKIl $\alpha$, and expressed as the percentage of the amount in control cells (mean \pm SD; ns, not significant; ${ }^{*} p=0.05,{ }^{* *} p=0.004$, Student's t test). C, HeLa cells transfected with GFP-CaMKII $\alpha$ and GFP-Abi1 were treated with or without $30 \mu \mathrm{m}$ ionomycin and $2 \mathrm{~mm} \mathrm{CaCl}$ for 10 min and examined by immunoblotting with indicated antibodies. Changes in P-CaMKIl $\alpha$ were quantified and expressed in arbitrary units as changes in P-CaMKII $\alpha /$ GFP-CaMKIl $\alpha$ compared with control cells. $D$, HeLa cells were transfected with GFP-CaMKII $\alpha$ with or without GFP-Abi1 or SEP-NR2B. Cells were treated with or without $30 \mu \mathrm{m}$ ionomycin for $10 \mathrm{~min}$ and immunoblotted with indicated antibodies.

$83-114), 44.1 \%$ of the amino acids are identical to those in the regulatory domain (amino acids 273-306) of CaMKII $\alpha$ (Fig. 2A). Homology of this nature has also been reported in NR2B, an NMDA receptor subunit (8.8\% identity) (Bayer et al., 2001), and in the Drosophila Eag potassium channel (11.8\% identity) (Wang et al., 2002; Sun et al., 2004). Binding of CaMKII $\alpha$ to this region in NR2B (amino acids 1290-1315) is implemented as a mechanism in which NR2B association retains CaMKII $\alpha$ activity even after dissociation of $\mathrm{Ca}^{2+} / \mathrm{CaM}$ (Bayer et al., 2001). This mechanism has also been reported for Drosophila Eag potassium chan- nel (Wang et al., 2002; Sun et al., 2004). One notable difference between Abil and these molecules is that Abil has high homology in the CaM-binding region of CaMKII $\alpha$ in addition to the autoinhibitory region. Pull-down assays with GST-tSNARE deletions including and excluding the region of homology show that this region (amino acids 83-114) is essential for interaction with CaMKII $\alpha$ (Fig. 2B). Quantification of CaMKII $\alpha$ binding to GST deletion mutants shows similar binding affinity of mutants containing the region of homology.

Abil negatively regulates CaMKII $\alpha$ Thr 286 autophosphorylation in a $\mathrm{Ca}^{2+}$ dependent manner

Because NR2B and Eag channel affect CaMKII $\alpha$ activation, we investigated whether Abil also affects CaMKII $\alpha$ activation by examining the level of Thr 286 autophosphorylation, a hallmark of CaMKII $\alpha$ activation (Fig. $3 A$ ). When GFP-Abil was overexpressed with Flag-CaMKII $\alpha$, basal level Thr 286 P-CaMKII $\alpha$ was attenuated by $\sim 60 \%$. However, GFP-Abil $\Delta$ tSNARE, which does not bind to CaMKII $\alpha$, increased basal level of Thr 286 autophosphorylation by $70 \%$. Increasing levels of GFP-Abil expression with Flag-CaMKII $\alpha$ resulted in a gradual decrease in Thr 286 autophosphorylation (Fig. 3B). Thus, Abil and NR2B/Eag channel elicit an opposite effect on CaMKII $\alpha$ activation. To observe the effect of calcium on Abil inhibition of $\mathrm{P}-\mathrm{CaMKII} \alpha, 30 \mu \mathrm{M}$ ionomycin, a calcium ionophore to induce $\mathrm{Ca}^{2+}$ influx into cells, was treated for $15 \mathrm{~min}$ (Fig. 3C). The inhibitory effects of Abil on CaMKII $\alpha$ at basal states were relieved upon ionomycin treatment. To further confirm that binding of CaMKII $\alpha$ to Abil has a negative effect on P-CaMKII $\alpha$, we compared the effects of Abil and NR2B on P-CaMKII $\alpha$. NR2B has been reported to sustain CaMKII $\alpha$ activity through its region of homology (Bayer et al., 2001). As expected, expression with Abil decreased P-CaMKII $\alpha$ compared with control cells and expression with SEP-NR2B increased P-CaMKII $\alpha$ (Fig. $3 D$ ). In ionomycin-treated cells, cotransfection with SEP-NR2B slightly increased P-CaMKII $\alpha$ compared with control cells and GFP-Abil-expressing cells. Although both proteins, Abil and NR2B, have homology to CaMKII $\alpha$, they regulate CaMKII $\alpha$ in opposite ways. These results suggest that Abil may be a negative regulator of CaMKII $\alpha$ during basal states before an increase in intracellular calcium.

Next we used Abil shRNA to reduce Abil expression in HeLa cells and hippocampal neurons and to investigate the effects of Abil knockdown on P-CaMKII $\alpha$. First, the specificity of the Abil shRNA was tested in HeLa cells stably expressing scrambled shRNA (P1) or Abil shRNA (S3) by transfecting wild-type (GFP- 
A

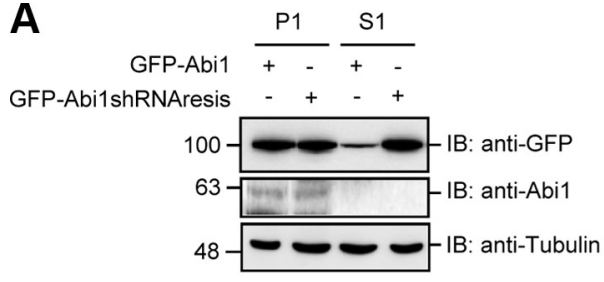

B

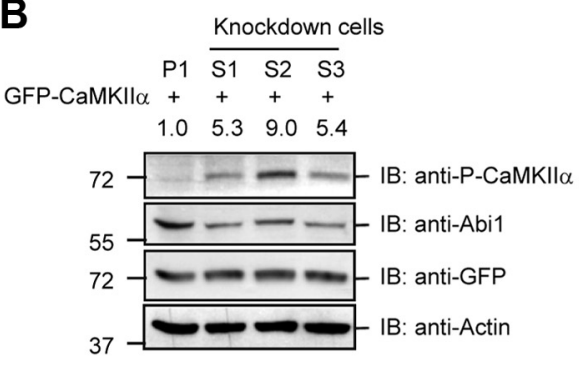

C
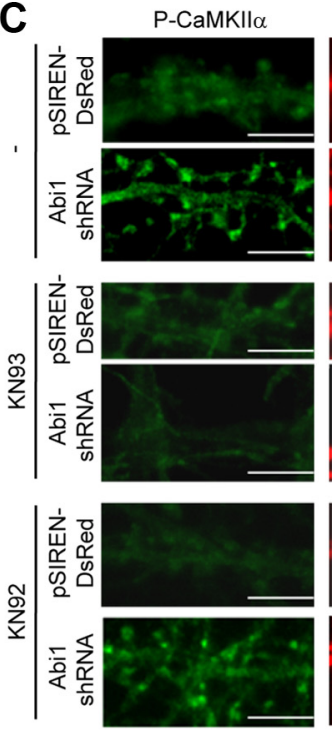
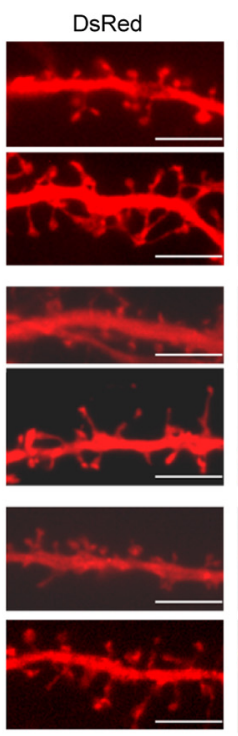
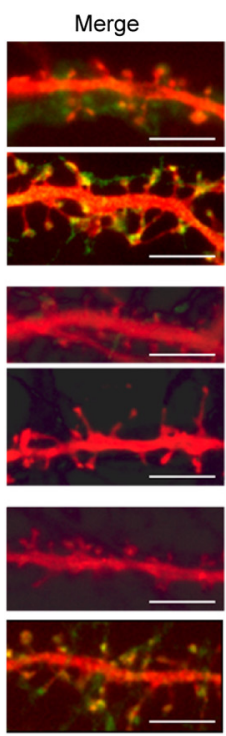

D

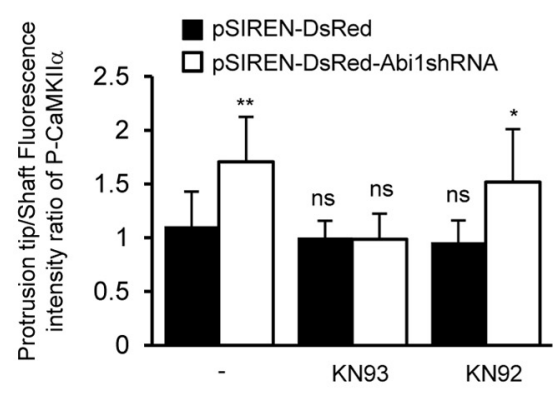

E

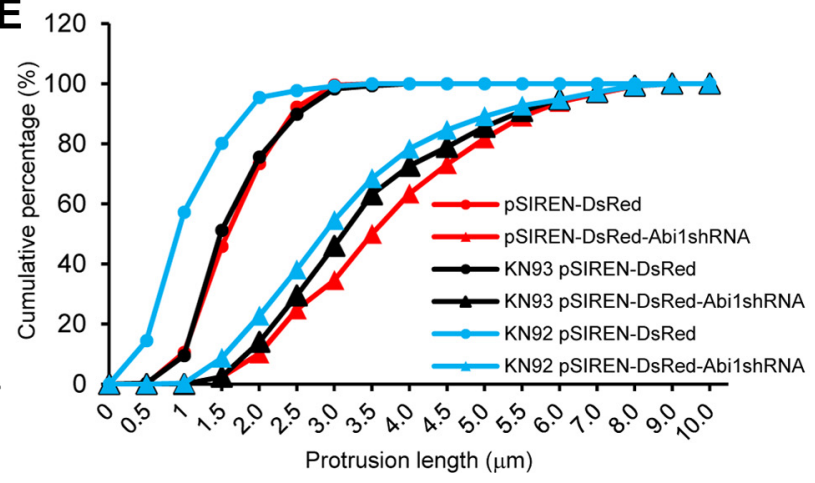

Figure 4. Knockdown of Abi1 in HeLa cells and rat hippocampal neurons elicit increased CaMKIll $\alpha$ autophosphorylation. $A$, HeLa cells stably expressing pSIREN-DsRed (P1) and pSIREN-DsRed Abi1shRNA (S1) were transfected with GFP-Abi1 or shRNA-resistant GFP-Abi1 (GFP-Abi1shRNAresis) and immunoblotted (IB) with indicated antibodies. Tubulin was used as the loading control. $\boldsymbol{B}$, HeLa cells stably expressing pSIREN-Dsred (P1) and pSIREN-DsRed Abi1shRNA (S1, S2, and S3) were transfected with GFP-CaMKII $\alpha$. CaMKII $\alpha$ autophosphorylation was examined by immunoblotting. Changes in P-CaMKII $\alpha$ were quantified and expressed in arbitrary units as changes in P-CaMKII $\alpha /$ GFP-CaMKIl $\alpha$ compared with control cells. C, 14 DIV neurons transfected with pSIREN-DsRed or p-SIREN-DsRed-Abi1shRNA (Abi1shRNA) were treated with or without $10 \mu \mathrm{m}$ KN93 or $10 \mu \mathrm{m}$ KN92. Representative neurites for each condition are shown. Scale bars, $5 \mu \mathrm{m}$. D, E, Results of subjecting 7310 dendritic protrusions from 120 neurons to morphometric analysis. Protrusion tip/shaft fluorescence intensity ratio of P-CaMKIl $\alpha$ in neurons was quantified for each condition (D). Compared with pSIREN-DsRed-transfected neurons, ratio of P-CaMKIl $\alpha$ increased in control and KN92-treated pSIREN-DsRed-Abi1shRNA neurons (mean \pm SEM, ${ }^{*} p=0.001$, ${ }^{* *} p<0.00001 ;$; 5 , not significant; Student's $t$ test). Protrusion lengths were measured for each condition $(\boldsymbol{E})$.

Abi1) or shRNA-resistant Abil (GFP-AbilshRNAresis) (Fig. $4 A)$. P1 cells show no change in expression of both GFP constructs, whereas S3 cells exhibit decreased expression of wildtype GFP-Abil but not GFP-AbilshRNAresis. Anti-Abil immunoblot shows that endogenous Abi1 is decreased in S3 cells in both cases. Using these cells, we demonstrate that knockdown of Abil in HeLa cells stably expressing pSIREN-DsRed-Abi1 shRNA increased Thr 286 autophosphorylation in CaMKII $\alpha$ compared with control cells (Fig. 4B). To observe these effects in neurons, 14 DIV rat hippocampal neurons transfected with pSIREN-DsRed and pSIREN-DsRed-Abil shRNA were treated with or without $10 \mu \mathrm{M}$ KN93, a CaMKII-specific inhibitor, or 10 mu KN92, an inactive compound of KN93 (Fig. 4C). Consistent with previously reported data (Proepper et al., 2007), Abil knockdown in 14 DIV hippocampal neurons showed immature dendritic spines and consist mostly of long filopodia. In control neurons, Thr 286 autophosphorylation in CaMKII $\alpha$ was present at low levels and dispersed throughout dendrites, but in Abi1knockdown neurons, the ratio of P-CaMKII $\alpha$ fluorescence intensity in protrusion tip to shaft was $\sim 60 \%$ increased, depicting a marked induction of P-CaMKII $\alpha$ in dendritic spines (Fig. $4 D$ ).
The increase of P-CaMKII $\alpha$ was reversed when treated with KN93 and unaffected by KN92 treatment. The localization of phospho-Thr $286 \mathrm{CaMKII} \alpha$ at the tips of the filopodial extensions is consistent with previous reports showing translocation of active CaMKII $\alpha$ to the synapse (Shen and Meyer, 1999; Bayer et al., 2001). In addition, length of protrusions did not change significantly after KN93 or KN92 treatment (Fig. 4E). Together, Abil negatively regulates CaMKII $\alpha$ activity in dendritic spines in $\mathrm{a} \mathrm{Ca}^{2+}$-dependent manner by binding to its catalytic domain.

\section{Abi1-CaMKII $\alpha$ interaction is modulated in a glutamate- dependent manner via CaM}

Because intracellular calcium modulates CaMKII $\alpha$ activity, and because increased intracellular calcium inhibits Thr 286 autophosphorylation by Abil (Fig. 3C), we investigated the effect of excitatory stimuli on CaMKII $\alpha$ and Abil interaction. Given that CaMKII $\alpha$ is known to be activated through glutamate receptor stimulation, the interaction of Abil and CaMKII $\alpha$ was examined in 21 DIV cortical neurons with glutamate and glycine, NMDA, and AMPA treatments. $\mathrm{KCl}$ and bicuculline were also used to induce depolarization of the neurons. Abil showed reduced 


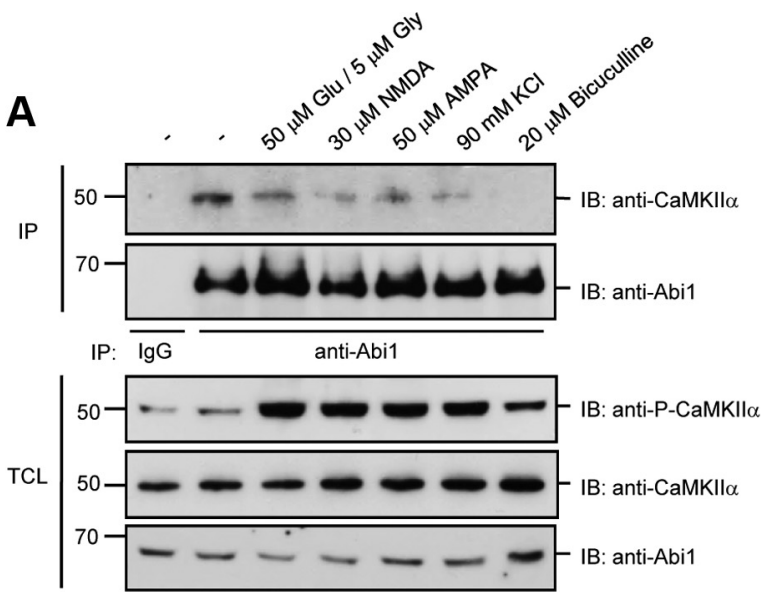

B
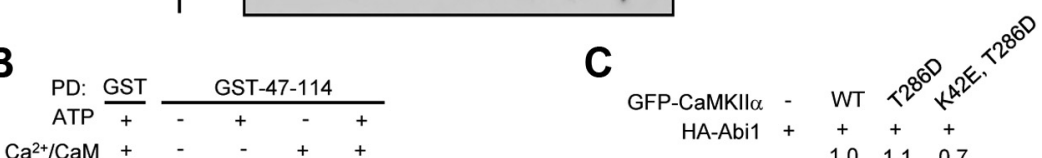
$\mathrm{Ca}^{2+} / \mathrm{CaM}+-\quad+\quad+$
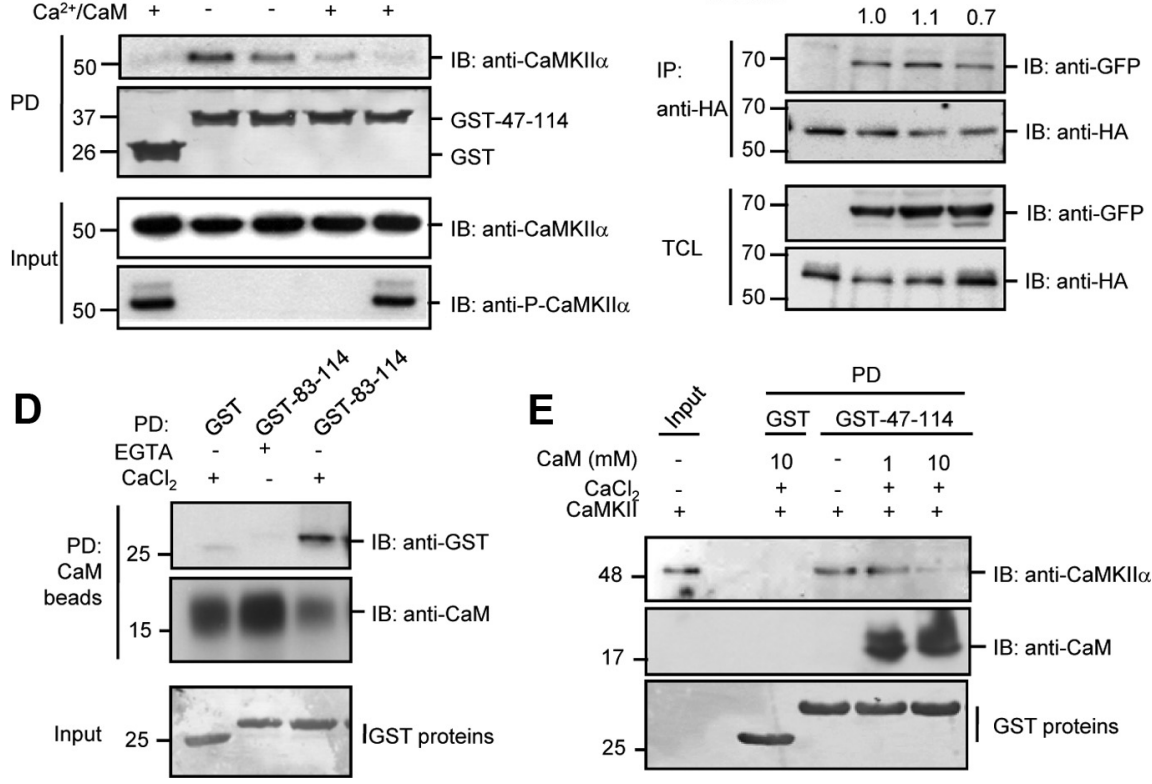

Figure 5. Interaction between Abi1 and CaMKIl $\alpha$ is disrupted by excitatory stimulation through $\mathrm{Ca}^{2+} / \mathrm{CaM}^{2}$ binding to Abi1. $\boldsymbol{A}$, 14 DIV rat cortical neurons were treated with $50 \mu \mathrm{m}$ glutamate and $5 \mu \mathrm{m}$ glycine for $5 \mathrm{~min}, 30 \mu \mathrm{m}$ NMDA for 5 min, $50 \mu \mathrm{M}$ AMPA for $5 \mathrm{~min}, 90 \mathrm{~mm} \mathrm{KCl}$ for $3 \mathrm{~min}$, or $20 \mu \mathrm{m}$ bicuculline for $30 \mathrm{~min}$. Lysates were immunoprecipitated (IP) with anti-Abi1 antibodies, subjected to SDS-PAGE, and then immunoblotted (IB) with anti-CaMKII $\alpha$ antibodies. B, Purified CaMKII was incubated with ATP and/or $0.3 \mathrm{~mm} \mathrm{Ca}{ }^{2+} / 2 \mathrm{~mm} \mathrm{CaM} \mathrm{at} 30^{\circ} \mathrm{C}$ for $10 \mathrm{~min}$, to promote activation of CaMKII, and then the purified GST or GST-Abi 1 tSNARE (GST-47-114) protein was added to the mixture and GST pull-down (PD) assays were performed. The interaction of Abi1 tSNARE with CaMKII was analyzed by immunoblotting with anti-CaMKII $\alpha$ antibodies. C, HeLa cells were cotransfected with HA-Abi1 and GFP-CaMKII $\alpha$ constructs (wild-type, autophosphorylation mimetic GFP-CaMKII $\alpha{ }^{\text {T286D }}$, or autophosphorylation mimetic kinasedead GFP-CaMKII $\alpha^{\text {K42E,T286D }}$, and the lysates were immunoprecipitated with anti-HA antibodies and immunoblotted with antiGFP antibodies. Ratio of bound GFP-CaMKIl $\alpha$ mutants to HA-Abi1 were quantified and expressed as arbitrary units compared with wild type. D, CaM Sepharose beads were used for pull-down of GST-83-114 in the presence of $1 \mathrm{~mm}$ EGTA or $2 \mathrm{~mm} \mathrm{CaCl}_{2}$. Pull-down assays were analyzed by Western blot as indicated. $\boldsymbol{E}, 1$ or $10 \mathrm{~mm} \mathrm{CaM}$, and $0.3 \mathrm{~mm} \mathrm{CaCl}$ were incubated with purified $\mathrm{CaMKII}_{\text {and }}$ GST-47-114. Binding of CaMKII and CaM to GST-47-114 was observed by immunoblotting with indicated antibodies. TCL, Total cell lysate.

binding affinity to CaMKII $\alpha$ after all the above stimulations (Fig. 5A).

To determine whether binding of Abil to CaMKII $\alpha$ is inhibited by CaM-dependent conformational change or by change in kinase activity, purified GST-Abil (amino acids 47-114) was incubated with purified rat CaMKII preincubated with or without $\mathrm{Ca}^{2+} / \mathrm{CaM}$, and/or ATP (Fig. $5 B$ ). In the presence of $\mathrm{Ca}^{2+} / \mathrm{CaM}$, interaction between CaMKII and GST-Abil (amino acids 47114 ) was significantly attenuated (Fig. 5B, lane 4 ) and completely abolished when CaMKII is autophosphorylated by the addition of ATP (Fig. $5 B$, lane 5). The binding of $\mathrm{Ca}^{2+} / \mathrm{CaM}$ to CaMKII $\alpha$ elicits three events: (1) a change in conformation where the catalytic domain is released from the inhibitory domain, (2) activation of the catalytic domain, and (3) last Thr 286 autophosphorylation (Lisman et al., 2002). So to confirm that Abi1-CaMKII $\alpha$ interaction is disrupted by CaM and not by the downstream effects of CaM binding to CaMKII $\alpha$, binding of Abil to GFP-CaMKII $\alpha^{\text {T286D }}$ (a mutant that mimics autophosphorylation and therefore has open conformation) and GFPCaMKII $\alpha^{\mathrm{K} 42 \mathrm{E}, \mathrm{T}^{286 \mathrm{D}} \text { (a mutant that has }}$ open conformation but lacking kinase activity) were examined (Fig. 5C). Compared with wild-type CaMKII $\alpha$, the mutants did not have significant differences in their interaction with Abil. These results indicate that $\mathrm{CaM}$ binding, and not alterations in conformation or kinase activity, prohibit Abil binding to CaMKII $\alpha$. Given that Abil has homology in the CaM-binding domain of CaMKII $\alpha$ (Fig. $2 A)$ and that the presence of $\mathrm{Ca}^{2+} / \mathrm{CaM}$ disrupts Abi1-CaMKII $\alpha$ interaction, we tested whether Abil can bind directly to CaM. Direct interaction of Abil (GST-83114) to $\mathrm{Ca}^{2+} / \mathrm{CaM}$ was observed in vitro (Fig. $5 D$ ) in the presence of calcium. In addition we confirmed the interaction with an in vitro competition assay where increasing amounts of $\mathrm{Ca}^{2+} / \mathrm{CaM}$ were added to GST47-114 and CaMKII. Binding of CaMKII and CaM to GST-47-114 were observed (Fig. 5E). Increasing amounts of $\mathrm{Ca}^{2+} / \mathrm{CaM}$ showed subsequent decrease in CaMKII binding to GST-47-114. Therefore, AbilCaMKII $\alpha$ interaction is regulated in a $\mathrm{Ca}^{2+} / \mathrm{CaM}$-dependent manner in which $\mathrm{Ca}^{2+} / \mathrm{CaM}$ binds to $\mathrm{Abil}$ and initiates dissociation of Abil from CaMKII $\alpha$.

\section{Phosphorylation of Abil tSNARE at Serine 88 by CaMKII $\alpha$ increases its affinity for $\mathrm{Ca}^{2+} / \mathrm{CaM}$}

Sequence analysis of Abil and CaMKII $\alpha$ demonstrates that Abil has a high degree of homology with the CaMKII $\alpha$ inhibitory domain, including sites of autophosphorylation (Fig. $2 A$ ). So we tested whether Abil is phosphorylated by CaMKII $\alpha$. Immunoprecipitation of phosphoserine proteins in 14 DIV cortical neuron lysates showed serine phosphorylation of Abil after treatment with 50 $\mu \mathrm{M}$ glutamate and $5 \mu \mathrm{M}$ glycine and this phosphorylation was not detected when $10 \mu \mathrm{M}$ KN93 was pretreated (Fig. 6A). There was no change in threonine phosphorylation of Abil under the same conditions (data not shown). These results suggest that activation of CaMKII $\alpha$ through the glutamate receptor may induce serine phosphorylation of Abil. Several mutants were generated using the 
GST-83-114 (amino acids 83-114 of Abi1) construct as the template and kinase assays were performed (Fig. 6B). Of the mutants, GST-R84E and GST-S88A showed ablation of phosphorylation, whereas GST-T96A and GST-TT113,114VA showed a slight decrease in phosphorylation. Since the binding of GST-R84E and GST-S88A to CaMKII $\alpha$ was not impaired (Fig. 6C), lack of phosphorylation does not seem due to inability of these mutants to interact with CaMKII $\alpha$. Lack of phosphorylation in GST-R84E suggests that this site may be needed as a part of CaMKII $\alpha$ phosphorylation consensus sequence $[\mathrm{RXX}(\mathrm{S} /$ T)]. Although Abil has homology with CaMKII $\alpha$ in three major autophosphorylation sites (Fig. 2A), Abil does not mimic phosphorylation in these amino acids, but instead Ser 88 of Abil seems to be a major phosphorylation site in the tSNARE domain by CaMKII $\alpha$. NR2B and Eag channel are also phosphorylated in the regions with homology with CaMKII $\alpha$ but, because they are phosphorylated in the serine that aligns with the Thr 286 autophosphorylation site, they differ from Abil phosphorylation (Bayer et al., 2001; Sun et al., 2004). To determine whether Ser 88 is the major site of Abil phosphorylation by CaMKII $\alpha$, we transfected HeLa cells with constitutively active GFP-CaMKII $\alpha^{\text {T286D }}$ and GFP-Abil or GFP-Abil $^{\text {S88A }}$ (Fig. 6D). GFP-Abi1 ${ }^{\text {S88A }}$ mutant shows a marked decrease in serine phosphorylation compared with GFP-Abi1, indicating that Ser 88 is a major site of phosphorylation by CaMKII $\alpha$.

Since Abil was found to bind to $\mathrm{Ca}^{2+} /$ $\mathrm{CaM}$, we investigated whether Abil phosphorylation changes its affinity for $\mathrm{Ca}^{2+}$ / CaM (Fig. 6E). Through pull-down assays with CaM Sepharose beads, we found that GFP-Abi1 ${ }^{\text {S88D }}$ mutant binds with higher affinity to $\mathrm{Ca}^{2+} / \mathrm{CaM}$ than wild-type and GFP-Abi1 ${ }^{\text {S88A }}$ mutant. This result suggests that Abil binds to $\mathrm{Ca}^{2+} / \mathrm{CaM}$ and that Ser 88 phosphorylation may regulate its binding affinity to $\mathrm{Ca}^{2+} / \mathrm{CaM}$.

\section{Abil serine 88 phosphorylation is essential for spine maturation via Rac pathway}

Abil is known to be essential for Rac-dependent regulation of actin dynamics through receptor tyrosine kinase pathways (Scita et al., 1999; Innocenti et al., 2004). Activated Rac GTPase in neurons, besides fostering the development and maintenance of dendritic spines through regulation of actin cytoskeleton (Tashiro and Yuste, 2004), increases dendritic branch stability, according to reports (Li et al., 2002). Hence, we investigated the effects of CaMKII $\alpha$ binding on Abil-dependent activation of Rac through pull-down assays with GST-PBD (p21-binding domain) (Fig. $7 A$ ). Rac activation by GFP-Abil overexpression was suppressed by cotransfection with Flag-CaMKII $\alpha$. These results suggest that the binding of Abil to CaMKII $\alpha$ sequesters Abil function to activate Rac. Next, we examined the effects of Abil Ser 88 phosphorylation

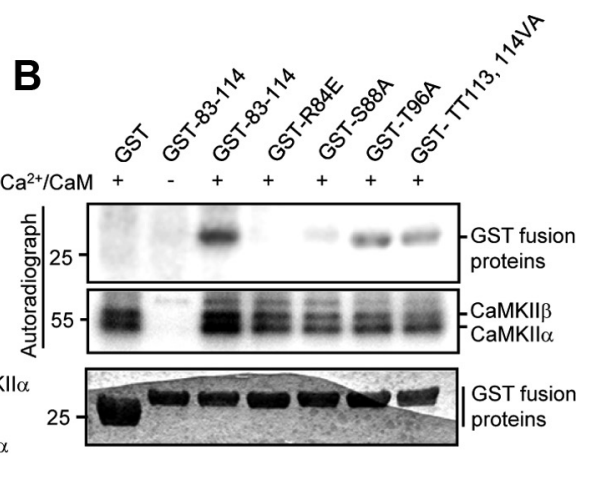

D

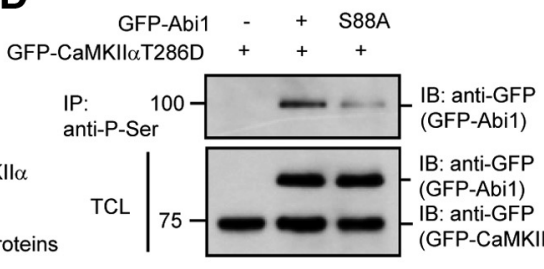
(GFP-CaMKIl $\alpha$ T286D)

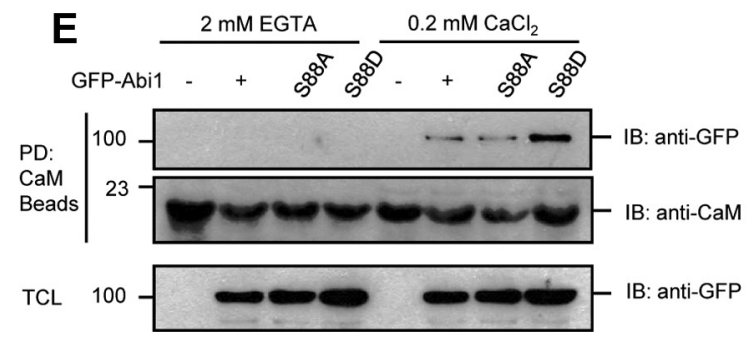

Figure 6. $\mathrm{Abi} 1$ is phosphorylated at serine 88 by CaMKIl $\alpha$ in vitro and in vivo and this phosphorylation enhances Abi1 binding for $30 \mathrm{~min}$. The lysates were immunoprecipitated (IP) with antiphosphoserine antibodies (anti-P-Ser) and immuand/or Ca ${ }^{+2} / \mathrm{CaM}$ at $30^{\circ} \mathrm{C}$ for 10 min to initiate CaMKII activation, and then aliquoted to samples containing wild-type (GST-8314 ) or mutant GST-tSNARE proteins (GST-R84E, GST-S88A, and GST-TT113,114VA) and incubated at $30^{\circ} \mathrm{C}$ for 30 min. Phosphor-

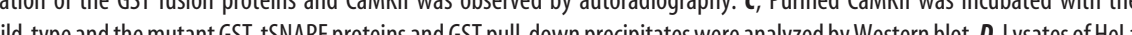
cells cotransfected with constitutively active GFP-CaMKII $\alpha^{\text {T286D }}$ and wild-type GFP-Abi1 or GFP-Abi1 ${ }^{\text {S88A }}$ were immunoprecipi$\mathrm{Abi}^{\mathrm{S}}{ }^{\mathrm{S} 8 \mathrm{~A}}$, or GFP-Abi1 ${ }^{\mathrm{S} 88 \mathrm{D}}$ were incubated with CaM Sepharose beads in the presence of $2 \mathrm{~mm} \mathrm{EGTA} \mathrm{or} 0.2 \mathrm{~mm} \mathrm{CaCl}_{2}$. Pull-down precipitates were subjected to SDS-PAGE and immunoblot with indicated antibodies. TCL, Total cell lysate.

on Abil-dependent Rac activation (Fig. 7B). Interestingly, Abildependent Rac activation was not observed in phosphorylationablated GFP-Abi1 ${ }^{\text {S88A }}$. In contrast, phosphorylation-mimicking GFP-Abi1 ${ }^{\text {S88D }}$ somewhat retained Rac-activating ability although with lower efficiency than wild-type Abil. To observe the effects of Abil phosphorylation in formation of dendritic spines, primary rat hippocampal neurons were transfected with the indicated constructs as shown in Figure 7C. GFP-Abil is localized specifically to spine heads as shown by the punctate staining, whereas GFP-Abil $\Delta \mathrm{tSNARE}$ is dispersed throughout neurite shafts and protrusions, indicating an essential role of the tSNARE domain for specific localization of Abil to spine heads. Previously, it was reported that Shank family proteins are crucial for trafficking of Abil to dendritic spines by interaction with Abi1 SH3 domain (Proepper et al., 2007). They showed that deletion of the Abi1 SH3 domain loses its postsynaptic localization and the mutant Abil is found only in the neurite shafts. Our study shows that the tSNARE domain of Abil is also important for its local- 

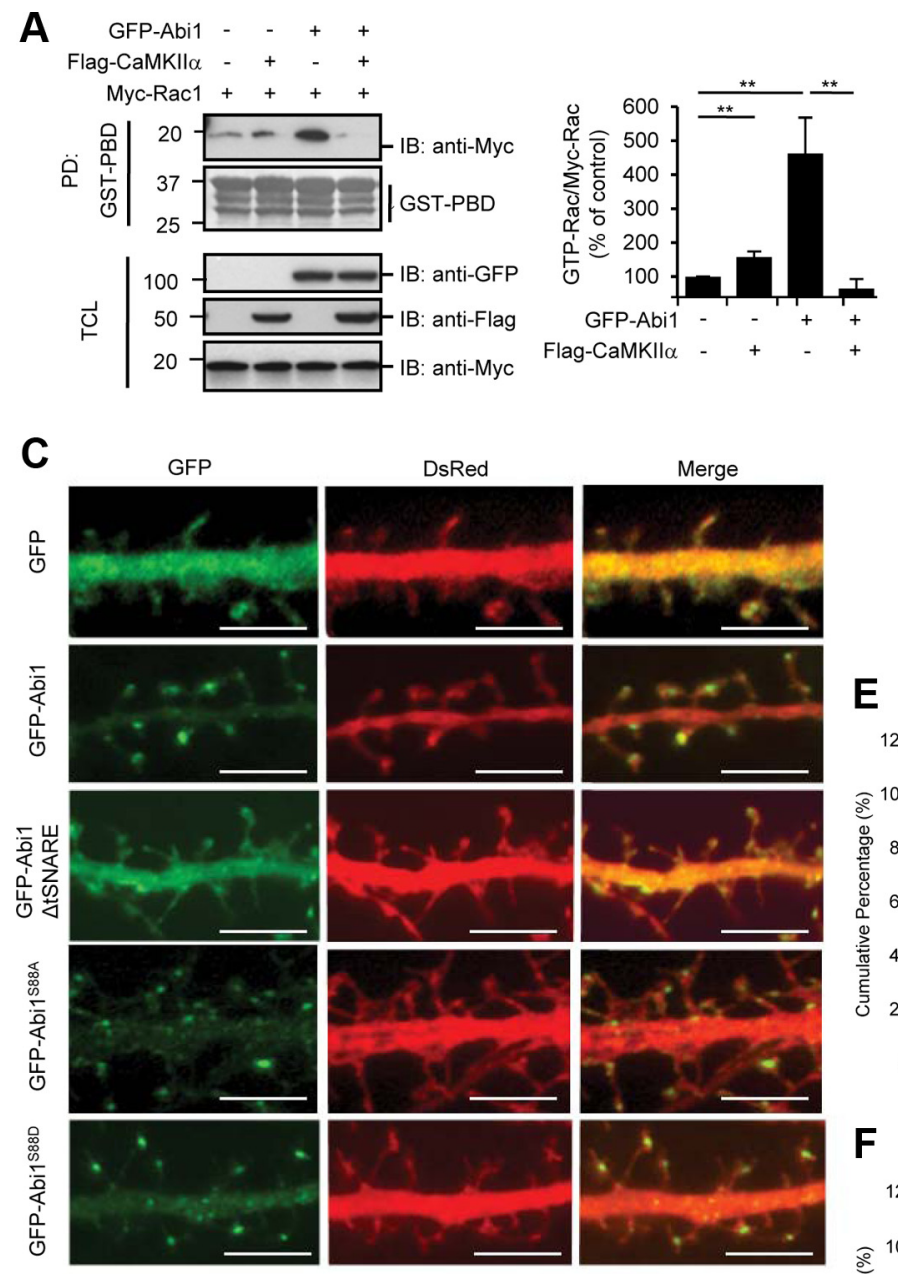

D

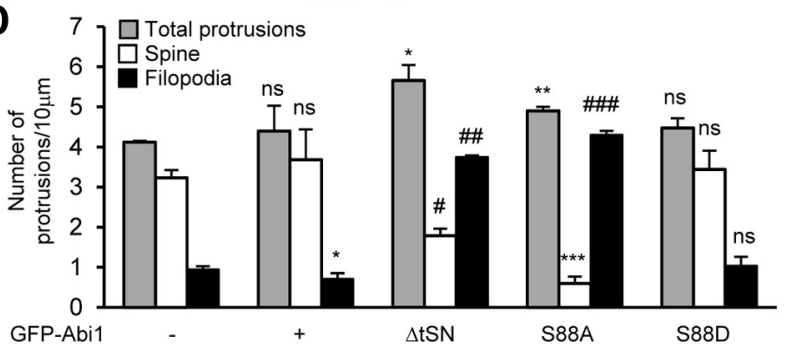

B
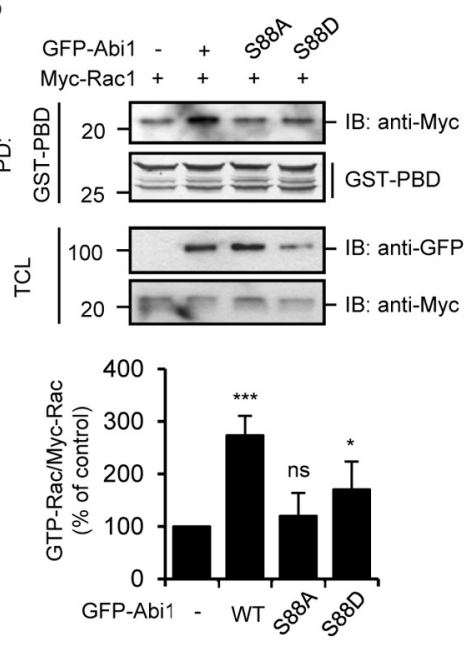

E

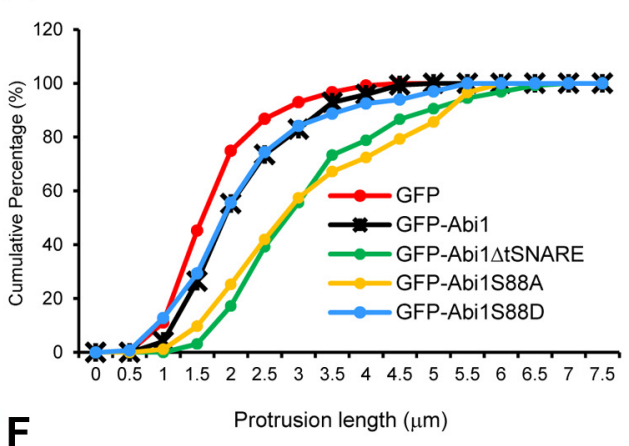

$\mathbf{F}$

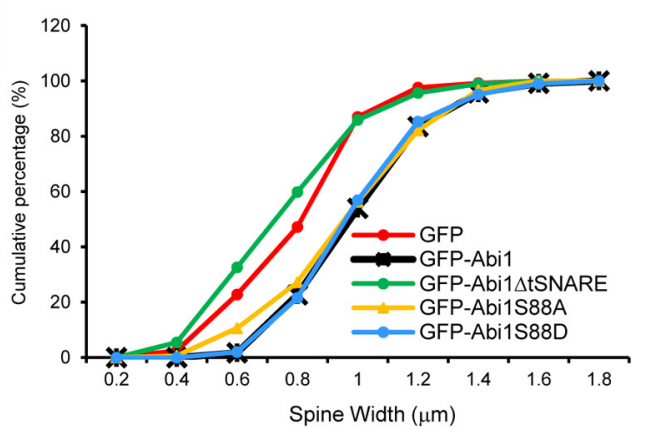

Figure 7. Phosphorylation of Abi1 at the serine 88 is critical for Rac activation and spine maturation. $\boldsymbol{A}$, HeLa cells were cotransfected with Flag-CaMKIl $\alpha$, Myc-Rac1, or GFP-Abi1 as indicated. $\boldsymbol{B}$, HeLa cells were cotransfected with Myc-Rac1 with either GFP-Abi1, GFP-Abi1 ${ }^{588 A}$, or GFP-Abi1 ${ }^{588 D}$. In $\boldsymbol{A}$ and $\boldsymbol{B}$, cell lysates were subjected to GST-PBD (p21-binding domain) pull-down (PD) and immunoblotted (IB) with anti-Myc antibodies. The ratio of GTP-Rac (bound Rac to GST-PBD)/Myc-Rac was quantified and expressed as the percentage of the amount in control cells from three independent experiments in both $\boldsymbol{A}$ and $\boldsymbol{B}$ (mean $\pm S D,{ }^{*} p=0.04,{ }^{* *} p=0.02,{ }^{* * *} p=0.007$; ns, not significant; Student's test). C, Rat hippocampal neurons were transfected with GFP, GFP-Abi1, GFP-Abi1 $\triangle$ tSNARE, GFP-Abi1 ${ }^{\text {S88A }}$, and GFP-Abi1 ${ }^{588 D}$ at 7 DIV and fixed at 14 DIV. DsRed was also coexpressed to show overall morphology of the neurons. Representative neurites for each condition are shown. Scale bars, $5 \mu \mathrm{m}$. $\boldsymbol{D}-\boldsymbol{F}$, Results after $150010 \mu \mathrm{m}$ dendritic segments from 100 neurons were subjected to morphometric analysis. $\boldsymbol{D}$, Number of total protrusions, spines, and filopodia per $10 \mu \mathrm{m}$ dendritic segments in hippocampal neurons transfected as indicated in C were quantified and compared with control cells using Student's $t$ test (mean \pm SEM, ${ }^{*} p=0.04$, ${ }^{* *} p=0.02$,

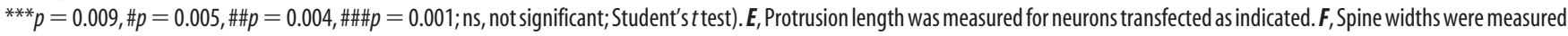
for neurons transfected as indicated. TCL, Total cell lysate.

ization to synapses. The tSNARE domain may be important for retention of Abil in synapses because GFP-Abil $\Delta$ tSNARE shows localization in both neurite shafts and spines. GFP-Abi1 ${ }^{\text {S88A }}$ shows punctate staining not only in dendritic spines but also in dendritic shafts. GFP-Abi1 ${ }^{\text {S88D }}$ has more pronounced punctate staining in dendritic spines, but also can be seen in the shafts as well. Morphometric analysis of neurons transfected as indicated in Figure $7 C$ shows changes in the number of protrusions, spines, and filopodia (Fig. 7D). Overexpression of GFP-Abil $\Delta$ tSNARE and GFP-Abi1 ${ }^{\mathrm{S} 88 \mathrm{~A}}$ in hippocampal neurons results in an increase in pro- trusions and also a change in number of spines and filopodia compared with GFP-transfected control neurons. In control neurons, the majority of protrusions were spines, but in GFP-

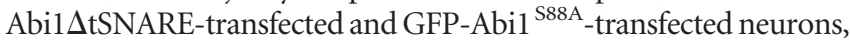
the majority of protrusions were filopodia. In addition, the protrusions in these neurons were longer (Fig. $7 E$ ). In contrast, overexpression of phosphorylation-mimicking GFP-Abi1 ${ }^{\mathrm{S} 88 \mathrm{D}}$ in neurons shows phenotype similar to overexpression of wild-type GFP-Abil (Fig. 7D,E). The increase in spine width as seen in GFP-Abil-overexpressed neurons was not affected by overex- 


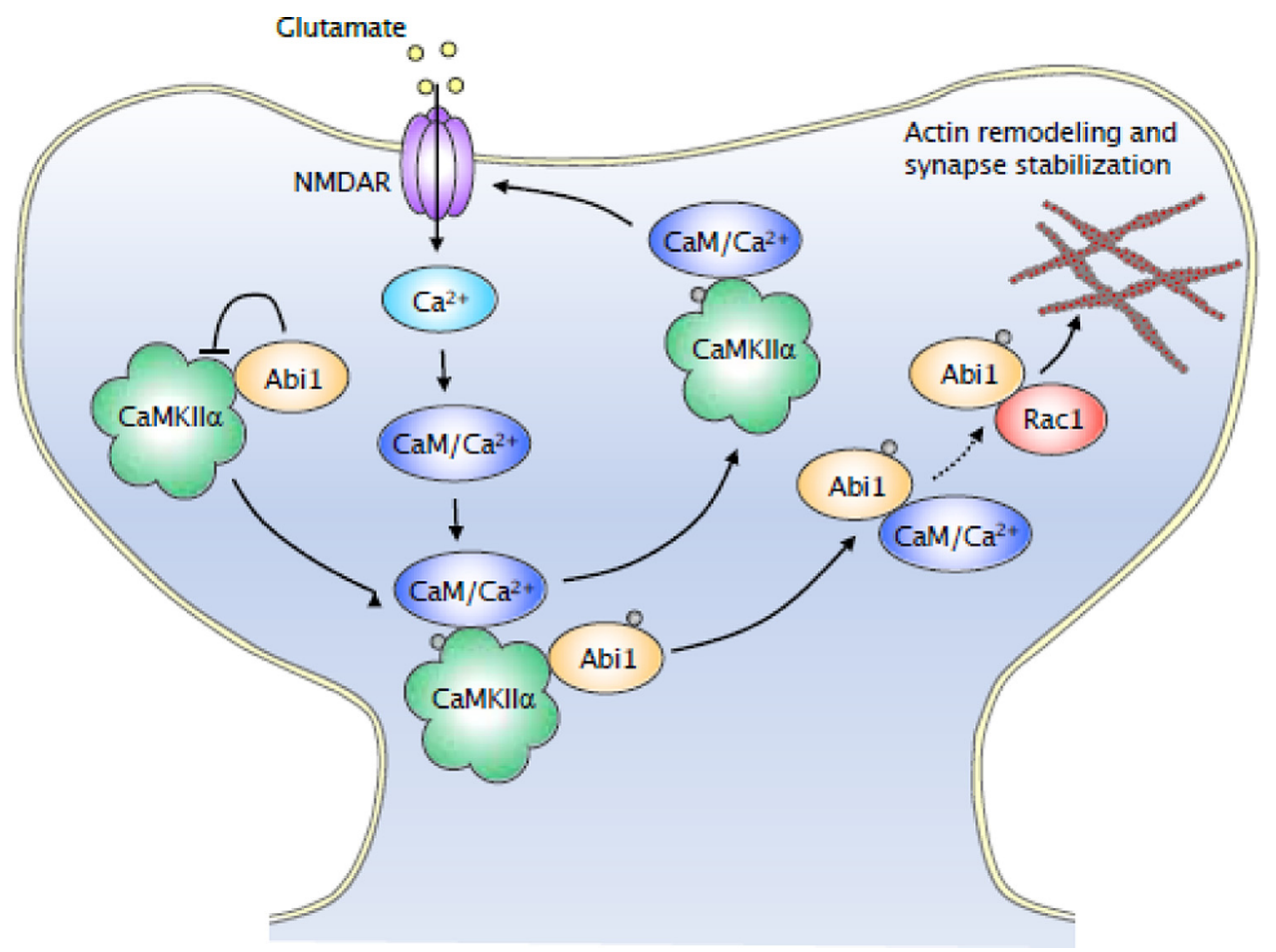

Figure 8. Proposed model for the roles of activity-dependent interaction between Abi1 and CaMKII $\alpha$ in spine regulation. At basal levels, Abi1 is bound to CaMKII and this interaction results in the mutual inhibition of their activity. Once glutamate receptors are activated, there is an increase in intracellular calcium, which induces $\mathrm{Ca}^{2+} / \mathrm{CaM}$ to trigger CaMKII activity. Active CaMKII phosphorylates Abi1 and binding of $\mathrm{Ca}^{2+} / \mathrm{CaM}$ to Abi1 elicits dissociation of Abi1 from CaMKII. Phosphorylated Abi1 then binds to Rac complex and activates Rac.

pression of phosphomutants GFP-Abi1 ${ }^{\text {S88A }}$ and GFP-Abi1 ${ }^{\text {S88D }}$ but was affected only by GFP-Abil $\Delta$ tSNARE (Fig. $7 F$ ). These results suggest that the tSNARE is essential for Abil's function to modulate spine morphology and that Abil phosphorylation at Ser 88 by CaMKII $\alpha$ plays a central role in this regulation.

\section{Discussion}

Regulation of CaMKII $\alpha$ activity is of substantial interest because of its implications in various areas such as LTP, LTD, experiencebased learning, memory, and structural plasticity (Silva et al., 1992; Maletic-Savatic et al., 1999; Toni et al., 1999; Lisman et al., 2002; Matsuzaki et al., 2004). In addition, model mice of neurological diseases were reported to have elevated levels of CaMKII activity or autophosphorylation. Ube3a maternal-deficient mice, a mouse model strain for Angelman syndrome, a disorder characterized by mental retardation and seizures, show increased levels of $\mathrm{P}-\mathrm{CaMKII} \alpha$ in the hippocampus (Weeber et al., 2003). Increased $\mathrm{P}$-CaMKII and activity was also observed in a mouse model for $\alpha$-thalassemia X-linked mental retardation (ATX-R) (Shioda et al., 2011). Our understanding of these neurological diseases, each of which are associated with abnormal dendritic spine formation, may benefit from these studies showing the importance of CaMKII $\alpha$ activity in spine regulation. However, the underlying molecular mechanisms are largely unknown. Abil-CaMKII $\alpha$ interaction provides a molecular mechanism in which CaMKII $\alpha$ activity may be regulated at basal states. Our results show that knockdown of Abil in hippocampal neurons results in an increase in CaMKII $\alpha$ Thr 286 autophosphorylation and that this upregulation coincides with the increase of P-CaMKII to the tips of dendritic protrusions (Fig. 4C). Dendritic protrusions in Abil-knockdown neurons are characterized as mostly long protrusions lacking stable mushroom-shaped spines (Proepper et al., 2007). Injection of active CaMKII $\alpha$ in hippocampal neurons also results in the formation of filopo- dia (Jourdain et al., 2003). The morphological changes seen in Abi1knockdown neurons are similar to the phenotypes of neurons with upregulated CaMKII $\alpha$ activity. It is possible that the changes in dendritic spine morphology related to CaMKII $\alpha$ activity may be due to disruption of its regulation by Abil.

Not only does Abil knockdown and GFP-Abil $\Delta$ tSNARE overexpression phenotype resemble activated CaMKII $\alpha$ in neurons, but also Rac activation. When the interaction between Abi1 and CaMKII $\alpha$ is blocked, Rac may become activated in an irregular manner, resulting in an increase of filopodial growth rather than spine enlargement. Compared with spine-specific localization of wild-type GFP-Abil, GFP-Abil $\Delta$ tSNARE is dispersed throughout the dendrite (Fig. 7C). Therefore, GFP-Abil $\Delta$ tSNARE may activate Rac throughout the length of the dendrite, leading to increased protrusion density rather than spine enlargement. Abil-CaMKII $\alpha$ interaction supports previous studies that demonstrate the importance of the proteins containing homology regions with $\mathrm{CaMKII} \alpha$ regulatory domain in the regulation of CaMKII $\alpha$ activity. Previous studies have reported channels, such as the Eag sodium channel and the NR2B, to have homology to CaMKII $\alpha$ regulatory domain (Bayer et al., 2001; Wang et al., 2002; Sun et al., 2004). The interaction of CaMKII $\alpha$ with these homology regions has been found to have a role in sustaining CaMKII $\alpha$ activity after glutamate stimulation. Because both the Eag sodium channel and NR2B are membrane proteins and abundant in synaptic membranes, they serve as anchors to sustain CaMKII $\alpha$ activation at the membranes after excitation. Similar to Eag sodium channel and NR2B, Abil binds to CaMKII $\alpha$ through a region with homology to the regulatory domain. On the other hand, as a soluble protein, Abil binds to CaMKII $\alpha$ and inhibits its kinase activity in the postsynaptic density before glutamate stimulation, which may serve as a mechanism ensuring stimulus-dependent spatiotemporal regulation of kinase activation. In addition, the three 
proteins-Eag sodium channel, NR2B, and Abi1-exhibit different modes of regulation by glutamate signaling. NR2B and Eag potassium channel bind to CaMKII $\alpha$ after glutamate signaling and enhance CaMKII $\alpha$ activity, while Abil binds to CaMKII $\alpha$ before glutamate signaling and inhibits CaMKII $\alpha$ activity. Last, the region of homology in Abil extends to the CaM-binding domain in CaMKII $\alpha$. On the contrary, NR2B and Eag channel exhibit little similarity to the CaM-binding domain (Fig. 2A). We demonstrate that through this region in Abil, Abil binds to CaM and this binding regulates Abi1-CaMKII $\alpha$ interaction. Not only does CaM bind to CaMKII $\alpha$ to initiate its kinase activity, but CaM also binds to Abi1 and disrupts its binding to CaMKII $\alpha$. Although CaMKII $\alpha$ homology regions in various proteins may have differences in regulating CaMKII $\alpha$ activity, having homology to the regulatory domain may serve as a guide for finding other endogenous regulators of CaMKII $\alpha$ activity.

Numerous studies show that CaMKII $\alpha$ is crucial for glutamatedependent structural plasticity (Yamasaki et al., 2008; Yamagata et al., 2009). In CaMKII $\alpha$ kinase-dead knock-in mice, tetanic stimulation failed to sustain spine enlargement, which is characteristic of tetanus-induced LTP (Yamagata et al., 2009). In addition, mice heterozygous for null mutation of CaMKII $\alpha$ display phenotypes similar to schizophrenia and other neurological diseases, such as bipolar disease (Yamasaki et al., 2008; Matsuo et al., 2009). These mice display immature dentate gyrus and severe deficits in working memory. However, specific mechanisms explaining the role of CaMKII $\alpha$ in modulating morphological changes in dendritic spines have not been defined. In addition, upstream signaling activating Rac in a glutamate-dependent manner has been poorly understood (Li et al., 2002; Tashiro and Yuste, 2004). Abil-CaMKII $\alpha$ interaction provides a molecular link in which glutamate stimulation regulates both Rac-dependent actin dynamics and also CaMKII $\alpha$ signaling. The interaction between the two proteins results in the dual inhibition of each other's function and this impediment is resolved after glutamate treatment, resulting in formation of $\mathrm{Ca}^{+2}$ / $\mathrm{CaM}$ that binds to both Abil and CaMKII $\alpha$ and in activation of Rac and CaMKII pathways.

We identified a phosphorylation site in Abil by CaMKII $\alpha$ that is crucial for Abil's function in dendritic spine regulation. Transfection of the phosphorylation-ablated Abil shows dendritic morphology similar to Abil knockdown neurons and neurons transfected with a tSNARE deletion mutant of Abil, which implies the significance of this phosphorylation in modulating spine morphology. Our results suggest that Abil phosphorylation by CaMKII $\alpha$ is critical for Rac activation, which in turn regulates actin dynamics in the spine. A previous report shows that Abil translocates to the nucleus within $1 \mathrm{~h}$ of $30 \mu \mathrm{M}$ NMDA treatment (Proepper et al., 2007). It would be interesting to see whether Abil Ser 88 phosphorylation has a role in nuclear translocation.

In this study, we discuss the role of Abil and CaMKII $\alpha$ in dendritic spines. However, Abil has also been reported to be targeted to growth cones and early synaptic contacts during neuronal development (Courtney et al., 2000; Proepper et al., 2007; Liebau et al., 2011). Since both structures also undergo $\mathrm{Ca}^{2+}$ dependent actin remodeling, the molecular mechanism we described pertaining to dendritic spines may also be applied to regulation of growth cones and early synaptic contacts. Although we observed a later stage of development, it would be interesting to see whether Abil-CaMKII $\alpha$ interaction could modulate actin dynamics at various stages of neuronal development.

In summary, Abil during basal conditions inhibits CaMKII $\alpha$ by binding to the kinase through its tSNARE domain, and this CaMKII $\alpha$ binding sequesters Abil-dependent Rac activation.
Glutamate receptor-mediated calcium influx induces $\mathrm{Ca}^{2+}$ / CaM-dependent activation of CaMKII $\alpha$, allowing Abil phosphorylation at Ser 88. Binding of $\mathrm{Ca}^{2+} / \mathrm{CaM}$ to Abil induces Abil dissociation from CaMKII $\alpha$, and phosphorylated Abil is able to activate the Rac pathway, initiating structural remodeling of dendritic spines. CaMKII $\alpha$ liberated from inhibition by Abil is activated and phosphorylates its numerous substrates in the spine (Fig. 8). Thus, Abil and CaMKII $\alpha$ may act synergistically to promote dynamic changes in dendritic spine morphology. We postulate that in dendritic spines the two proteins inhibit each other's function until excitatory signaling, such as activation of glutamate receptors, is initiated. Interaction of CaMKII $\alpha$ with Abil demonstrates a novel mechanism in which CaMKII $\alpha$ is inhibited by an intermolecular interaction through regions of homology, rather than an intramolecular mechanism through inhibitory domains of the holoenzyme. This interaction also makes it possible for Abil and CaMKII $\alpha$, two important regulators of synaptic plasticity, to keep each other in check until neuronal activation occurs. Furthermore, the interaction between these two proteins through the TSNARE domain of Abil implies a novel role of tSNARE domains in not only membrane fusion but also in signaling pathways. The findings in this study suggest that the activity-regulated interaction between Abil and CaMKII $\alpha$ may be a molecular mechanism underlying activity-dependent structural plasticity in dendritic spines.

\section{References}

Andersen R, Li Y, Resseguie M, Brenman JE (2005) Calcium/calmodulindependent protein kinase II alters structural plasticity and cytoskeletal dynamics in Drosophila. J Neurosci 25:8878-8888.

Bayer KU, De Koninck P, Leonard AS, Hell JW, Schulman H (2001) Interaction with the NMDA receptor locks CaMKII in an active conformation. Nature 411:801-805.

Chang S, De Camilli P (2001) Glutamate regulates actin-based motility in axonal filopodia. Nat Neurosci 4:787-793.

Cingolani LA, Goda Y (2008) Actin in action: the interplay between the actin cytoskeleton and synaptic efficacy. Nat Rev Neurosci 9:344-356.

Courtney KD, Grove M, Vandongen H, Vandongen A, LaMantia AS, Pendergast AM (2000) Localization and phosphorylation of Abl-interactor proteins, Abi-1 and Abi-2, in the developing nervous system. Mol Cell Neurosci 16:244-257.

Dillon C, Goda Y (2005) The actin cytoskeleton: integrating form and function at the synapse. Annu Rev Neurosci 28:25-55.

Dubielecka PM, Cui P, Xiong X, Hossain S, Heck S, Angelov L, Kotula L (2010) Differential regulation of macropinocytosis by Abil/Hssh3bp1 isoforms. PloS One 5:e10430.

Echarri A, Lai MJ, Robinson MR, Pendergast AM (2004) Abl interactor 1 (Abi-1) wave-binding and SNARE domains regulate its nucleocytoplasmic shuttling, lamellipodium localization, and wave-1 levels. Mol Cell Biol 24:4979-4993.

Innocenti M, Frittoli E, Ponzanelli I, Falck JR, Brachmann SM, Di Fiore PP, Scita G (2003) Phosphoinositide 3-kinase activates Rac by entering in a complex with Eps8, Abi1, and Sos-1. J Cell Biol 160:17-23.

Innocenti M, Zucconi A, Disanza A, Frittoli E, Areces LB, Steffen A, Stradal TE, Di Fiore PP, Carlier MF, Scita G (2004) Abil is essential for the formation and activation of a WAVE2 signalling complex. Nat Cell Biol 6:319-327.

Jourdain P, Fukunaga K, Muller D (2003) Calcium/calmodulin-dependent protein kinase II contributes to activity-dependent filopodia growth and spine formation. J Neurosci 23:10645-10649.

Leng Y, Zhang J, Badour K, Arpaia E, Freeman S, Cheung P, Siu M, Siminovitch K (2005) Abelson-interactor-1 promotes WAVE2 membrane translocation and Abelson-mediated tyrosine phosphorylation required for WAVE2 activation. Proc Natl Acad Sci U S A 102:1098-1103.

Li Z, Aizenman CD, Cline HT (2002) Regulation of rho GTPases by crosstalk and neuronal activity in vivo. Neuron 33:741-750.

Liebau S, Steinestel J, Linta L, Kleger A, Storch A, Schoen M, Steinestel K, Proepper C, Bockmann J, Schmeisser MJ, Boeckers TM (2011) An SK3 
channel/nWASP/Abi-1 complex is involved in early neurogenesis. PloS One 6:e18148.

Lisman J, Schulman H, Cline H (2002) The molecular basis of CaMKII function in synaptic and behavioural memory. Nat Rev Neurosci 3:175-190.

Maletic-Savatic M, Malinow R, Svoboda K (1999) Rapid dendritic morphogenesis in CA1 hippocampal dendrites induced by synaptic activity. Science 283:1923-1927.

Matsuo N, Yamasaki N, Ohira K, Takao K, Toyama K, Eguchi M, Yamaguchi S, Miyakawa T (2009) Neural activity changes underlying the working memory deficit in alpha-CaMKII heterozygous knockout mice. Front Behav Neurosci 3:20.

Matsuzaki M, Honkura N, Ellis-Davies GC, Kasai H (2004) Structural basis of long-term potentiation in single dendritic spines. Nature 429:761-766.

Nimchinsky EA, Sabatini BL, Svoboda K (2002) Structure and function of dendritic spines. Annu Rev Physiol 64:313-353.

Proepper C, Johannsen S, Liebau S, Dahl J, Vaida B, Bockmann J, Kreutz MR, Gundelfinger ED, Boeckers TM (2007) Abelson interacting protein 1 (Abi-1) is essential for dendrite morphogenesis and synapse formation. EMBO J 26:1397-1409.

Rosenberg OS, Deindl S, Sung RJ, Nairn AC, Kuriyan J (2005) Structure of the autoinhibited kinase domain of CaMKII and SAXS analysis of the holoenzyme. Cell 123:849-860.

Ryan XP, Alldritt J, Svenningsson P, Allen PB, Wu GY, Nairn AC, Greengard $P$ (2005) The Rho-specific GEF Lfc interacts with neurabin and spinophilin to regulate dendritic spine morphology. Neuron 47:85-100.

Scita G, Nordstrom J, Carbone R, Tenca P, Giardina G, Gutkind S, Bjarnegård M, Betsholtz C, Di Fiore PP (1999) EPS8 and E3B1 transduce signals from Ras to Rac. Nature 401:290-293.

Shen K, Meyer T (1999) Dynamic control of CaMKII translocation and localization in hippocampal neurons by NMDA receptor stimulation. Science 284:162-166.

Shioda N, Beppu H, Fukuda T, Li E, Kitajima I, Fukunaga K (2011) Aberrant calcium/calmodulin-dependent protein kinase II (CaMKII) activity is associated with abnormal dendritic spine morphology in the ATRX mutant mouse brain. J Neurosci 31:346-358.
Silva AJ, Stevens CF, Tonegawa S, Wang Y (1992) Deficient hippocampal long-term potentiation in alpha-calcium-calmodulin kinase II mutant mice. Science 257:201-206.

Sun XX, Hodge JJ, Zhou Y, Nguyen M, Griffith LC (2004) The eag potassium channel binds and locally activates calcium/calmodulin-dependent protein kinase II. J Biol Chem 279:10206-10214.

Tashiro A, Yuste R (2004) Regulation of dendritic spine motility and stability by Racl and Rho kinase: evidence for two forms of spine motility. Mol Cell Neurosci 26:429-440.

Toni N, Buchs PA, Nikonenko I, Bron CR, Muller D (1999) LTP promotes formation of multiple spine synapses between a single axon terminal and a dendrite. Nature 402:421-425.

Wang Z, Wilson GF, Griffith LC (2002) Calcium/calmodulin-dependent protein kinase II phosphorylates and regulates the Drosophila eag potassium channel. J Biol Chem 277:24022-24029.

Weeber EJ, Jiang YH, Elgersma Y, Varga AW, Carrasquillo Y, Brown SE, Christian JM, Mirnikjoo B, Silva A, Beaudet AL, Sweatt JD (2003) Derangements of hippocampal calcium/calmodulin-dependent protein kinase II in a mouse model for Angelman mental retardation syndrome. J Neurosci 23:2634-2644.

Wu GY, Cline HT (1998) Stabilization of dendritic arbor structure in vivo by CaMKII. Science 279:222-226.

Yamagata Y, Kobayashi S, Umeda T, Inoue A, Sakagami H, Fukaya M, Watanabe M, Hatanaka N, Totsuka M, Yagi T, Obata K, Imoto K, Yanagawa Y, Manabe T, Okabe S (2009) Kinase-dead knock-in mouse reveals an essential role of kinase activity of $\mathrm{Ca}^{2+} /$ calmodulin-dependent protein kinase II $\alpha$ in dendritic spine enlargement, long-term potentiation, and learning. J Neurosci 29:7607-7618.

Yamasaki N, Maekawa M, Kobayashi K, Kajii Y, Maeda J, Soma M, Takao K, Tanda K, Ohira K, Toyama K, Kanzaki K, Fukunaga K, Sudo Y, Ichinose H, Ikeda M, Iwata N, Ozaki N, Suzuki H, Higuchi M, Suhara T, et al. (2008) Alpha-CaMKII deficiency causes immature dentate gyrus, a novel candidate endophenotype of psychiatric disorders. Mol Brain 1:6.

Zhang H, Macara IG (2006) The polarity protein PAR-3 and TIAM1 cooperate in dendritic spine morphogenesis. Nat Cell Biol 8:227-237. 